\title{
REGULATION OF CHARLATANS IN HIGH-SKILL PROFESSIONS
}

\author{
Jonathan B. Berk \\ Jules H. van Binsbergen \\ Working Paper 23696 \\ http://www.nber.org/papers/w23696 \\ NATIONAL BUREAU OF ECONOMIC RESEARCH \\ 1050 Massachusetts Avenue \\ Cambridge, MA 02138 \\ August 2017
}

We thank Svetlana Bryzgalova, Peter DeMarzo, Vincent Glode, Peter Kondor, Christian Opp, Paul Pfleiderer, Amit Seru, Gregor Matvos and seminar participants at LBS (Academic Symposium), UCLA and Rice University for helpful comments. The views expressed herein are those of the authors and do not necessarily reflect the views of the National Bureau of Economic Research.

NBER working papers are circulated for discussion and comment purposes. They have not been peer-reviewed or been subject to the review by the NBER Board of Directors that accompanies official NBER publications.

(C) 2017 by Jonathan B. Berk and Jules H. van Binsbergen. All rights reserved. Short sections of text, not to exceed two paragraphs, may be quoted without explicit permission provided that full credit, including $(\subset$ notice, is given to the source. 
Regulation of Charlatans in High-Skill Professions

Jonathan B. Berk and Jules H. van Binsbergen

NBER Working Paper No. 23696

August 2017

JEL No. D11,D18,G18,K2,K22,K23,L51

\begin{abstract}
We model a market for a skill that is in short supply and high demand, where the presence of charlatans (professionals who sell a service that they do not deliver on) is an equilibrium outcome. We use this model to evaluate the standards and disclosure requirements that exist in these markets. We show that reducing the number of charlatans through regulation decreases consumer surplus. Although both standards and disclosure drive charlatans out of the market, consumers are worse off because of the resulting reduction in competition amongst producers. Producers, on the other hand, strictly benefit from the regulation, implying that the regulation we observe in these markets likely derives from producer interests. Using these insights, we study the factors that drive the cross-sectional variation in charlatans across professions. Professions with weak trade groups, skills in larger supply, shorter training periods and less informative signals regarding the professional's skill, are more likely to feature charlatans.
\end{abstract}

Jonathan B. Berk

Stanford University

Graduate School of Business

655 Knight Way

Stanford, CA 94306

and NBER

jonathan.b.berk@gmail.com

Jules H. van Binsbergen

The Wharton School

University of Pennsylvania

3620 Locust Walk

Philadelphia, PA 19104

and NBER

julesv@wharton.upenn.edu 
"We write today to ask what specific steps FINRA will take to address the pervasive misconduct among its financial advisers and to protect families looking to invest their hard-earned savings." U.S. Senators E. Warren and T. Cotton, Letter to FINRA May 11th 2016.

An important role for government in the modern economy concerns the regulation of standards and information disclosure for goods and services. As the introductory quote from Senators Warren and Cotton illustrates, the justification legislators use for this role is generally the protection of consumers. Indeed, the idea that regulation is in consumers' interests is often taken for granted. Consequently, when Egan, Matvos and Seru (2016) documented that in the financial services sector $7 \%$ of active financial advisors have a misconduct conviction or settlement on their record, the results stirred enough interest to spur the congressional hearing that resulted in the letter to FINRA quoted from above.

In this paper we question the conventional wisdom that the regulation of information disclosure and standards benefits consumers. We show that for professions that require a skill in short supply that is in great demand, government regulation through disclosure requirements or standards reduces consumer surplus. Instead, the primary effect of regulation is to make producers better off, providing an explanation for why trade groups often lobby for and are in favor of high standards.

We study the role of regulation in a parsimonious equilibrium model that features charlatans - agents that know that they do not possess the skill to offer a particular service but nevertheless may optimally choose to enter that profession and offer the service. In the model, skilled agents are in short supply and consumers learn about the skillfulness of the professional through a signal. The main intuition for why regulation reduces consumer surplus is that, in expectation, consumers get what they pay for. That is, before any government intervention, the probability that the consumer could be dealing with a charlatan is reflected in a lower price for the service. If the government imposes a standard by requiring a lower bound on the providers' signal (a license), the price of the service or good increases to reflect the lower probability of consumers having to deal with a charlatan. If this were the only effect, consumers would be left indifferent: the increase in their expected benefit is perfectly offset by the higher price that has to be paid for the service. However, there is an additional effect. The minimum standard reduces the number of providers, thereby reducing competition in the market. This reduced competition increases prices further, and thus lowers consumer surplus.

Perhaps most surprisingly, our results extend to information disclosure requirements. 
It is often argued that informing consumers better about the products they buy, leaves them better off, because they can make better choices. ${ }^{1}$ However, this logic ignores the equilibrium effect on prices that such information revelation has. Consider the case where the government is perfectly informed about who the charlatans are, and instead of setting standards, simply communicates this information to consumers. It is tempting to conclude that by providing this information, the government will make consumers better off. We prove the opposite. Even though the information fully drives charlatans out of the market, consumers are left worse off. The reason is that once again, there are two price adjustments that follow from such information revelation. First, prices of the remaining professionals will go up to reflect that consumers now have a zero probability of dealing with a charlatan. This leaves consumers indifferent, they pay more, but they get better service. But second, because competition amongst providers is reduced, prices will go up further, and this second effect reduces consumer surplus.

Although overall welfare within a sector (that is the total welfare of all producers and consumers within the sector) is rarely cited by policy makers as the reason to regulate, one might argue that maximizing overall welfare within the sector should be the policy objective. However, even under this objective, it is difficult to justify regulation. Information disclosure, because it allows consumers to better discriminate, increases the supply of skilled workers and thus increases overall welfare. But as we have already pointed out, disclosure also increases producer welfare through higher wages. Thus producer interest are aligned with overall welfare, so one would not expect there to be much need to require information disclosure. Producers should disclose themselves. Standards, however, have the opposite effect. Introducing a minimum standard into a market that does not have a minimum standard reduces overall welfare because it reduces the supply of skill. So regulation in this case is undesirable. Thus, in both cases, if the objective is to maximize overall welfare within the sector, there appears little need to regulate.

These insights also have important implications for the debate in the economics literature on certification and licensing. ${ }^{2}$ The difference between these two regulations is that a professional cannot practice without a license, but can practice without being certified. Consequently, we can interpret a licensing requirement as a minimum standard and certification as requiring information disclosure. The existing literature comes down in favor of certification because it provides consumers with information they otherwise do not have access to. It can thus help them disentangle charlatans from skilled professionals

\footnotetext{
${ }^{1}$ Examples that comes to mind are the regulation of accounting standards and derivatives markets.

${ }^{2}$ See Kleiner and Krueger (2013) and Kleiner (2000) and the references therein.
} 
while not reducing competition by inadvertently denying licenses to skilled professionals. Although the observation that certification reduces competition less than licensing is correct, the argument misses the fact that certification also reduces competition and as a result it also reduces consumer surplus. In contrast, if the objective of the regulation is to help professionals by increasing their wages, then licensing is preferred over certification. After all, licensing both discloses information and further decreases competition by not allowing some skilled workers to practice. In summary, in our setting, certification is never preferred, not by producers nor by consumers.

Our results are particularly important in light of the increase in the level of licensing (and certification) in the modern economy. ${ }^{3}$ For example, Hogan (1983) documents that the second half of the 20th century has seen an explosive growth in licensing. As of 1950, 73 occupations were licensed in one or more states, with 13 occupations requiring a license in every jurisdiction (Council of State Governments, 1952). Twenty years later (according to a 1969 Department of Labor study (USDOL)), for all professional and occupational groups almost 5,000 different licenses are granted across the country, covering more than 500 occupations and averaging out to 100 per state. There is also large cross-sectional variation across states, both in the number and the type of professions that require a license. In terms of numbers, California and Illinois are the leading regulators, licensing more than 175 occupations each.

Another important feature of the data is that there is large variation across professions in the number of professionals convicted of misconduct as well as the standards imposed. For example, Egan et al. (2016) document that in the financial services sector, $7 \%$ of active financial advisors have a misconduct conviction or settlement on their record. That paper also documents that about half of those advisors are fired, and about half of the ones fired find a new job in financial services. The median settlement amount for misconduct is $\$ 40,000$. Contrast this to the medical profession. In medicine, Studdert et al (2016) find that a very small fraction of doctors (1\%) are responsible for most malpractice settlements (32\%), yet very few of those doctors are fired. In 2015 out of 931,921 licensed doctors in the US, only 290 doctors (0.03\%) had their license revoked, and even temporary suspensions (706) are rare $(0.075 \%) .{ }^{4}$ These facts are surprising given what is at stake. A total of $32 \%$ of the 66,426 paid claims in the authors' data set involved the death of the patient

\footnotetext{
${ }^{3}$ Even homeopaths and psychic readers have certification boards. One Chief Examiner of The National Certifying and Testing Board of the American Federation of Certified Psychics and Mediums Inc. is specialized in pet communication. On a more existential note, one wonders why a psychic examiner would even need to administer an examination to determine whether a candidate is qualified.

${ }^{4}$ Source: Federation of State Medical Boards: U.S. Medical Regulatory Trends and Actions.
} 
(21,509 patients) and an additional 53\% involved either a major physical injury (15\%) or a significant (but not major) physical injury (38\%). Further, the financial implications are also very large: the median settlement amount was over $\$ 200,000$. Another stark difference between medicine and finance are the standards required to practice. An implication of the high wages of people who work in the financial sector is that their skill is apparently in very short supply. Yet in most states to become a financial advisor requires little more than a high school education and the ability to pass an exam. To practice medicine, on the other hand, requires at least 10 years of post high school education and usually two different sets of board examinations. In both professions the exams themselves do not appear to test the required skills directly — even highly skilled practitioners cannot pass the exams without studying for them.

The main insight from our analysis is that regulation of professionals is in the interests of professionals not consumers. So, to understand this cross-sectional variation in regulation, one has to understand the tradeoffs that skilled professionals face in lobbying for regulation. They trade off the benefit of reducing the number of charlatans against the cost of a standard that catches some unlucky skilled workers. We use our model to better understand these tradeoffs. The entry decision of charlatans (as well as the resulting equilibrium wage in the profession), are based on (1) the opportunity cost required to enter the profession, (2) the amount of uncertainty surrounding the professional's ability to offer the service or not, and (3) the degree to which the skill the professional offers is in short supply. As such the model provides a simple characterization of the parameters that describe which professions are likely to have charlatans. Professions with weak trade groups, skills in larger supply, shorter training periods and less informative signals regarding the professional's skill, are more likely to feature charlatans.

Our results also suggest that the malpractice conviction rate, which depends on the standard that determines the definition of malpractice, while receiving a lot of attention from regulators and the press, is not particularly informative about the number of charlatans in a profession. More importantly, it is uninformative about the value a particular profession offers to consumers. If one is interested in the variation in charlatans across professions, wages are an important market-based indicator of the number of charlatans. In our model, all else equal, professions with larger cross-sectional variation in wages have more charlatans than professions with a low cross-sectional variation.

A key assumption in our model is that agents are either born with the capacity to acquire the skill or they are not. Because the skill is in short supply and great demand, 
this implies that in a market that does not feature charlatans, all agents who possess the capacity to acquire the skill will acquire it. So the number of agents that have the skill is fixed and is unaffected by market prices. That is, given that our interest is in professions that require a skill in short supply (such as finance and medicine), the second best alternative for those professionals is so inferior that they all choose to work in the sector. However, even in professions in which this assumption does not hold, given the substantial training periods involved in most high-skilled jobs, it is reasonable to assume that the short-run elasticity of labor supply with respect to wages is essentially zero. Whereas consumers can instantly react to changes in licensing and certification, the labor supply response to wages (in professions like accounting) can take many years to materialize. Thus, the assumption that the supply of skilled workers is invariant to wage changes holds in the short term even in professions in which some agents with the capacity to acquire the skill choose not to acquire it.

Finally, our paper is related to the literature on standards spurred by the seminal contribution of Leland (1979), who derives a model with a continuum of goods of different quality in which the government is fully informed and consumers have no information on the quality of the good. As a consequence, the subsequent literature based on the model in Leland (1979) does not feature charlatans: producers who claim to offer the service but do not. This difference is crucial because in that model when standards are introduced, the resulting price rise spurs the entry of higher quality goods, something that cannot happen in our model. In our model price rise spurs the entry of charlatans, but that does not raise quality. This key difference provides Leland's main result, that standards can sometimes benefit consumers. Another difference is that we study a homogeneous good that is bought by heterogeneous consumers, whereas Leland (1979) studies heterogenous goods that are bought by homogeneous (uninformed) consumers. As a consequence, Leland (1979) studies how the supply of actual product quality changes with regulation, not how the entry of charlatans is affected by regulation.

\section{Data}

We begin by providing empirical evidence substantiating the claim in the introduction that there is wide variation in standards and conviction rates across professions. Table 1 presents data for five professions: financial advisors, medical doctors, lawyers, real estate agents and accountants. Our main data sources are as follows. For financial advisors, we 


\begin{tabular}{llll} 
Panel A & & & \\
\hline Profession & Education & Passing Rate & With settle/convict \\
\hline Financial Advisors & $<1$ & $65 \%$ (Series 7) $72 \%$ (Series 66) & $7 \%$ \\
Medical Doctors & $>8$ & $94 \%$ (S1), 95\% (S2), 98\% (S3) & $6 \%$ \\
Lawyers & 7 & $59 \%$ & No Data \\
Real Estate Agents & $<2$ & $50-60 \%$ & No Data \\
Accountants & $>4$ & $47 \%$ & No Data \\
\hline
\end{tabular}

\begin{tabular}{llll} 
Panel B & \multicolumn{3}{l}{} \\
\hline Profession & License revoked/disbarred & Fired after settle/convict & Damages \\
\hline Financial Advisors & $\approx 0 \%$ & $50 \%$ & $\$ 40,000$ \\
Medical Doctors & $0.03 \%$ & Low & $\$ 204,750$ \\
Lawyers & $0.10 \%$ & Low & $\$ 30,000-\$ 50,000$ \\
Real Estate Agents & $0.15 \%$ & No Data & No Data \\
Accountants & $0.10 \%$ & Low & $\$ 50,000-\$ 100,000$ \\
\hline
\end{tabular}

Table 1: Comparison of Charlatan Statistics Across Professions:The table presents data for four professions: financial advisors, medical doctors, lawyers and real estate agents. Column 1 lists the profession. Column 2 and 3 list training characteristics. Column 2 lists the number of years since high school required to work in the profession. Column 3 lists the passing rate of the licensing exams required to work in the profession. Column 5 lists the total number of active professionals with a conviction/settlement for malpractice or misconduct on their record as a fraction of the total number of professionals active in that profession. Column 6 lists the number of professionals whose license are revoked or are disbarred from their profession as a fraction of total number of professionals. Column 7 lists the number of professionals that are fired after a conviction/settlement for misconduct or malpractice as a fraction of the number of professionals that settle or receive a conviction. The last column list the median damages after settlement or conviction.

rely on the work by Egan et al. (2016). For medical doctors we rely on the data presented in Studdert, Bismark, Mello, Singh and Spittal (2016) as well the data presented by the Federation of State Medical Boards. For lawyers we use data presented by Kritzer and Vidmar (2015). For real estate agents, we rely on data provided by Nguyen-Chyung and Shelef (2016) and for accountants we use data from the American Institute of Certified Public Accountants (CPA).

The second column of Panel A lists the amount of training required to practice as a professional (measured as the number of years of post high school training). To become a financial advisor a college education is not formally required. Obtaining a license is relatively easy, and in many cases only requires product knowledge. For medical doctors on the other hand, it requires four years of college, four years of medical school, a residency and several more years to become a specialist. For lawyers, it requires a college education and three years of law school. For real estate agents very little formal education is required, and for accountants a college education and in some cases a master's degree suffices. Next, we compare standards that are imposed through various exams in the third column of 
Panel A. The table lists the passing rates of these exams. For financial advisors, the passing rates of the Series 7 and Series 66 exam were $65 \%$ and $72 \%$ respectively in 2015 . These rates are low relative to the passing rates of the medical board exams. These board exams consist of three steps (S1,S2 and S3), and the national passing rates are close to $100 \%$ for all three steps. The standard for lawyers is imposed through the bar exam, which had a national average passing rate of $59 \%$ in 2015 . To become a real estate agent, states impose a real estate license examination, which have passing rates between $50 \%$ and $60 \%$. Finally, for accountants, the passing rate of the CPA exam is the lowest of all professions and on average (across the four subject areas) equals $47 \%$.

The fourth column of Panel A lists the number of professionals in a given profession that have a settlement or conviction for malpractice/misconduct on their record. Across all professions, settlements vastly outnumber convictions. The number is similar for financial advisors (7\%) and medical doctors (6\%). While anecdotal evidence suggests that for lawyers the number is as high or higher, very little data is available as insurance companies of lawyers do not release data and many settlements are kept confidential (Kritzer and Vidmar (2015)). The second column in Panel B lists the number of professionals whose license was revoked or were disbarred in 2015 as a fraction of the total number of active professionals. The number is the lowest for medical doctors and the highest for real estate agents.

The third column in Panel B summarizes the action of employers when their employees settle or are convicted for misconduct or malpractice. For financial advisors the number of employees who are fired is high and equal to $50 \%$. For the other professions we have data on, this number is low. Most doctors are in fact not employed by the hospital they work in. Consequently, the only corrective action the hospital can take is to revoke the admitting privileges of the doctors, which happens rarely. Anecdotal evidence for lawyers suggests that settlements for malpractice cases are viewed as a cost of doing business and therefore do not often result in the firing of the lawyer. In addition, lawyers at large law firms are insured for such events. The last column of Panel B lists the median damages for settlements/convictions. The number is by far the largest for medicine, which is not surprising given what is at stake in many malpractice cases.

Anecdotally, most people would probably agree that there are few charlatan doctors and many more charlatan finance professionals. More definitively, Berk and van Binsbergen (2015) find that more than half of the mutual funds in their sample are managed 
by managers who destroy value. ${ }^{5}$ Because managers can always avoid destroying value by indexing their capital, the fact that these managers choose not to do this suggests that they choose to actively trade to mimic skilled managers and thus not reveal, in their trading, that they lack skill.

It is difficult to make any definitive claims on the prevalence of charlatans from the evidence in Table 1. Yet, the evidence that is there is at least consistent with the medical profession having far fewer charlatans than the finance profession. First note that the vast majority of doctors are allowed to keep practicing even after multiple malpractice convictions, suggesting that a malpractice conviction might have more to do with the difficulty of the procedure than the doctor being a charlatan. In addition pass rate on the medical boards is extremely high, suggesting that very few charlatans make it through 11 years of medical school. In contract, in finance $25 \%$ of financial advisors leave the profession after a conviction. The required time to train is much shorter and the training does not appear to prepare candidates for the required examinations. The pass rate on the required examinations is much lower than medicine, suggesting that many unqualified people attempt the exam. In short, the circumstantial evidence is that medicine is a profession of high standards and few charlatans, while finance is a profession of lower standards and more charlatans.

Notice that the fraction of doctors and financial advisors with a malpractice or misconduct record is quite similar, suggesting that conviction rates are not a reliable measure of the prevalence of charlatans. Lawyers have a much shorter training period than doctors and the bar exam for lawyers is much tougher to pass given the training, which suggests that the profession should have a significant number of charlatans, yet like medicine, few lawyers are disbarred. So it is unclear what the prevalence of charlatans in the legal profession is.

If indeed, as the circumstantial evidence suggests, there are many more charlatans in finance than in medicine, it is worth asking why standards in finance are not raised to eliminate the charlatans as appears to be the case in medicine. That is, is it in the interests of consumers, as Senators Warren and Cotton assume, to eliminate charlatans by raising standards or disclosing information? In the next section, we present a develop a model to help answer this important policy question.

\footnotetext{
${ }^{5}$ The reason that paper finds that, on average, mutual fund managers add a substantial amount of money is that the skilled managers manage most of the money. Consistent with the assumptions in this paper, investors correctly infer the probability of the manager being a charlatan and thus invest most of their money with skilled managers.
} 


\section{The Model}

Our objective in this paper is to study a labor market that features professionals who have a skill that is in very short supply and large demand. Because the resulting wages are so high, the profession is very attractive to producers. Thus, all agents who have this skill choose to produce and, in addition, agents who do not have the skill also choose to offer the service. Formally, our world consists of a unit measure of unskilled agents, $c \in C$, where $C=[0,1]$, who consume a product or service that requires a skill in short supply. In addition, a measure $\lambda$ of agents exist who have a skill that allows them to produce this product or service. The unskilled agents can pose as skilled agents, what we term "charlatans." A charlatan cannot produce the product or service so he or she confers no benefit. If an agent chooses to provide the product of the skill (whether skilled or not) he earns a wage that is paid by consumers which will be determined in equilibrium. If the agent chooses not to provide the service, she earns a reservation wage which we normalize to zero. All agents know whether they are skilled or not.

The unskilled agents in our model are also consumers, so the measure of potential consumers is 1 (we assume, for simplicity that skilled agents can provide the service to themselves at no cost). That there are more potential producers than potential consumers captures the idea that with no barriers to entry, charlatans will "flood" the market. If an agent chooses to consume, then he must find a single producer who will provide the product or service. A producer can only service one consumer.

Consumers value the service or product differently. Let $b(c):[0,1] \rightarrow[0,1]$ be the value each consumer assigns to the service. That is, $b(c)$ is consumer $c$ 's willingness to pay (the maximum amount she is willing to pay). Consumers are ordered such that $b^{\prime}(c) \geq 0$. Our focus is a market for a skill that is valued by many consumers but is in very short supply. Therefore we assume throughout that $\lambda$ is small and $b^{\prime \prime}(c) \leq 0$. The concavity of the $b$ function reflects that the skill in short supply is highly valued by a large number of consumers. Consumers maximize their expected payoff from consuming the product or service that results from the skill, that is, they choose which producer to use by maximizing the expected benefits minus the costs (the wage paid).

To fix ideas, it is worth briefly describing what happens in the equilibrium when consumers can observe whether a producer is skilled. In this case there are only $\lambda$ producers (all of which are equally skilled), so only $\lambda$ consumers can consume. Wages must therefore equilibrate to clear the market. Thus, the equilibrium wage that all (identical) skilled 
workers make, is $b(1-\lambda)$. Clearly, no producer has an incentive to lower the wage. Any producer that raises the wage will find no customers. Notice that even though the skill is in short supply, most customers who actually consume the service have positive consumer surplus, that is, they pay less than their willingness to pay. As the supply of skill decreases, consumer surplus decreases.

When skill is unobservable, it is straightforward to show that unless agents have some information about whether a producer is actually skilled, an extreme lemon's problem exists. More accurately, when consumers have no information on the skill level of producers, the resulting equilibrium features a wage of zero. The reason is that for any positive wage, all agents choose to produce (offer the service), implying that the supply of the skill is $1+\lambda$, which is strictly larger than the demand of 1 , implying that wages cannot be positive. With zero wages, both skilled workers and charlatans are indifferent about producing, so a continuum of equilibria exists. In equilibria where skilled workers choose not to provide the service, charlatans are the providers of the skill, so consumers who choose to "consume" get what they pay for - they pay nothing and get nothing. In equilibria that feature skilled producers, consumers pay nothing but get something, implying that consumer surplus is positive.

To allow for a market that features charlatans and positive wages in equilibrium, we provide consumers with information. Specifically, we assume that every producer can choose to generate a signal $\eta$, that is observable to all agents, and is given by

$$
\eta \equiv q+\tilde{u}
$$

where $q$ is equal to 1 if the agent is skilled and zero otherwise, and $u$ is a normally distributed mean zero random variable with standard deviation $\sigma$. Denote the c.d.f. of the signal produced by skilled and unskilled agents as $F_{s}(\eta)$ and $F_{u}(\eta)$ respectively, with associated density functions $f_{s}(\eta)$ and $f_{u}(\eta)$. Note that $\frac{f_{s}}{f_{u}}$ is strictly increasing, that is, the monotone likelihood ratio property holds.

We will say an unskilled agent chooses to become a charlatan if she chooses to generate a signal. Let $y$ be the fraction of unskilled agents that choose to become charlatans. Given 
$y$, the probability that the agent is skilled given a signal $\eta$ is,

$$
\begin{aligned}
\pi(\eta) & \equiv P[\text { skilled } \mid \eta]=P[\eta \mid \text { skilled }] \frac{P[\text { skilled }]}{P[\eta]} \\
& =f_{s}(\eta) \frac{\frac{\lambda}{y+\lambda}}{f_{s}(\eta) \frac{\lambda}{y+\lambda}+f_{u}(\eta) \frac{y}{y+\lambda}} \\
& =\frac{f_{s}(\eta)}{f_{s}(\eta)+f_{u}(\eta)\left(\frac{y}{\lambda}\right)} .
\end{aligned}
$$

Because we will need it repeatedly, it makes sense to compute $\pi^{\prime}(\eta)$ explicitly and note that it is positive $(\pi(\eta)$ is strictly increasing):

$$
\pi^{\prime}(\eta)=\left(\frac{y}{\lambda}\right) \frac{f_{u}(\eta) f_{s}^{\prime}(\eta)-f_{u}^{\prime}(\eta) f_{s}(\eta)}{\left(f_{s}(\eta)+f_{u}(\eta)\left(\frac{y}{\lambda}\right)\right)^{2}}>0
$$

because the fact that $\frac{f_{s}(\eta)}{f_{u}(\eta)}$ is strictly increasing implies $f_{u}(\eta) f_{s}^{\prime}(\eta)-f_{u}^{\prime}(\eta) f_{s}(\eta)>0$.

The timing is as follows. Agents first decide if they are going to generate a signal by comparing the expected wage of producing to their reservation wage. Because their reservation wage at this point is zero, they will generate a signal so long as expected wages are positive. One can think of the signal as the product of an apprenticeship program (like a medical residency) or a grade on a required examination. Given a signal, agents then decide if they still want to produce. Because attending university or undertaking an apprenticeship program might require permanently giving up other opportunities, we allow for the possibility that agents face an opportunity cost. Specifically, if, after generating the signal, the agent chooses not to produce, his reservation wage drops by $A \geq 0$. That is, because the reservation wage is normalized to zero initially, it is $-A$ once the signal is generated. Thus, once the signal is generated, agents will choose to produce so long as their wage, given their signal realization, exceeds their reservation wage, $-A .^{6}$

Once the signal is generated, agents decide if they want to consume. They do this by finding the producer with the signal that maximizes their payoff, that is, consumers solve

$$
\begin{aligned}
\max _{\eta} & (b(c) \pi(\eta)-w(\eta)) \\
\text { s.t. } & b(c) \pi(\eta)-w(\eta) \geq 0 .
\end{aligned}
$$

\footnotetext{
${ }^{6}$ One could also add a cost of the initial signal to the model. The results remain qualitatively the same, but at the cost of increased complexity.
} 
For simplicity we will adopt a short-hand formulation and will say that consumer $c$ consumes signal $\eta$ when we mean consumer $c$ chooses to use the product or service produced by the producer that generated signal $\eta$. Let $\eta(c):[0,1] \rightarrow[-\infty, \infty]$ be the solution to this problem, that is, the signal $\eta$ that consumer $c$ chooses to consume. Then define the inverse function $c(\eta) \equiv \eta^{-1}(\eta)$, that is, the consumer $c$ who chooses to consume a signal $\eta{ }^{7}$

An equilibrium (which we more formally define in Appendix A) is a wage function, $w(\eta)$ and a consumption function, $c(\eta)$, such that

- all skilled agents choose to obtain a signal, and $0 \leq y \leq 1$ fraction of unskilled agents choose to obtain a signal (become charlatans),

- agents with signals that exceed $\bar{\eta}$ choose to produce,

- consumers choose which signal to consume by maximizing the benefit of consuming (i.e., solve the maximization problem (3)) and

- markets clear - every producer who chooses to produce given their signal realization has a consumer willing to consume from her.

Proposition 7 in Appendix A derives the conditions under which an equilibrium of the model exists and characterizes the equilibrium. So long as the opportunity cost $A$ is not too large, an equilibrium in which at least some unskilled agents and all skilled agents choose to produce, exists. All equilibria feature a strictly positive fraction of producers who are charlatans. Once the signal is revealed, only producers with a signal that exceeds $\bar{\eta}$ choose to produce.

To understand, intuitively, how the equilibrium consumption and wage functions are derived, note that the market clearing condition requires that the number of consumers consuming signals below $\eta$ equals the number of producers with signals below $\eta$ who choose to produce, that is,

$$
\int_{\bar{\eta}}^{\eta} \lambda f_{s}(x)+y f_{u}(x) d x=\int_{\bar{\eta}}^{\eta} d c(x) .
$$

Taking derivatives in (4) gives:

$$
c^{\prime}(\eta)=\lambda f_{s}(\eta)+y f_{u}(\eta) .
$$

\footnotetext{
${ }^{7}$ If $b^{\prime}(c)>0$, then $\eta^{\prime}(c)>0$ and so $c(\eta)$ is well defined and also strictly increasing.
} 
Integrating provides the function

$$
\begin{aligned}
c(\eta) & =\lambda F_{s}(\eta)+y F_{u}(\eta)-\lambda+1-y \\
& =1-\lambda\left(1-F_{s}(\eta)\right)-y\left(1-F_{u}(\eta)\right)
\end{aligned}
$$

where the constant of integration is determined by the boundary condition that the consumer with the largest $b$ chooses to consume the largest signal, that is, that $c(\infty)=1$. There are measure $1+\lambda$ potential producers but only measure 1 consumers, so $\bar{\eta}$ is determined in equilibrium to ensure that demand equals supply. That is, the measure of potential producers who choose not to produce equals $\lambda$ :

$$
\lambda F_{s}(\bar{\eta})+y F_{u}(\bar{\eta})=\lambda
$$

Equilibrium wages are determined from the consumer choice problem, that is, (3). The solution, $\eta(c)$, to this problem, assuming it exists, solves the first order condition

$$
w^{\prime}(\eta)=b(c) \pi^{\prime}(\eta)
$$

Recall that $c(\cdot)=\eta^{-1}(\cdot)$, so (6) can be rewritten as:

$$
w^{\prime}(\eta)=b(c(\eta)) \pi^{\prime}(\eta)
$$

Integrating (7) gives

$$
w(\eta)=\int_{\bar{\eta}}^{\eta} b(c(v)) \pi^{\prime}(v) d v-A
$$

where the constant of integration is determined by the condition that $w(\bar{\eta})=-A$, that is, a producer with signal $\bar{\eta}$ must be indifferent between supplying the skill or working for the reservation wage. Producers who get a signal that is less than $\bar{\eta}$ earn less than $-A$ and thus choose not to produce and instead work for the reservation wage of $-A$.

To explore how competition sets wages, integrate (8) by parts to give

$$
w(\eta)=b(c(\eta)) \pi(\eta)-\int_{\bar{\eta}}^{\eta} b^{\prime}(c(v)) c^{\prime}(v) \pi(v) d v-A
$$

because $b(c(\bar{\eta}))=b(0)=0$. Simplifying using (1) and (5) provides

$$
w(\eta)=b(c(\eta)) \pi(\eta)-\lambda \int_{\bar{\eta}}^{\eta} b^{\prime}(c(v)) f_{s}(v) d v-A
$$


The first term is the expected benefit derived from consuming the skill. If the skill provider with signal $\eta$ was a monopolist he would capture all the rents and this would be her wage. Because there is competition in this model, wages are lowered by the next two terms. The second term is the total marginal benefit delivered by all skilled producers with a lower signal than $\eta$. Because producers with higher signals than $\eta$ earn higher wages, they have no incentive to undercut a producer with signal $\eta$. So a producer with signal $\eta$ only faces competition from producers with lower signals. By lowering wages by this term, a producer with signal $\eta$ ensures that other producers with lower signals will not undercut her. This term therefore captures the effect on wages of competition from other producers. The last term ensures that agents with signals below $\bar{\eta}$ will choose not to produce, so it captures the effect of potential competition from producers that are not in the market. That is, wages are lower by $A$ to prevent too many producers from entering thereby ensuring that demand and supply are equilibrated.

All equilibria feature charlatans. For most parameter values that satisfy the existence conditions, all unskilled agents choose to become charlatans $(y=1)$, but there are cases in which the unskilled agents are indifferent between becoming a charlatan or not, so in these cases they mix and so a fraction $1-\lambda \leq y<1$ of unskilled agents choose to become charlatans. To understand where the lower bound on $y$ comes from, first note that it is possible for the market to break down completely. To see how this can happen, make $A$ large enough so that the expected wage of charlatans is negative. In this case, no unskilled agent will choose to become a charlatan, so consumers know that the equilibrium does not feature charlatans. The wage for all producers then jumps to the wage in the equilibrium without charlatans, $b(1-\lambda)$. But at this (high) wage, the expected wage for a charlatan is positive, so unskilled agents will then choose to become charlatans. Thus no equilibrium exists. Proposition 7 in Appendix A derives the existence conditions which then implies the lower bound on $y$. Note that equilibria always exist when $A=0$.

\section{Regulation of Information}

It is often taken for granted by regulators (and consumer protection groups) that disclo-

sure benefits consumers. The reason regulators make this assumption is that disclosure increases the information of consumers allowing them to make better choices. Unfortunately, in our context, this reasoning is incorrect. In fact, the opposite result is true. Disclosure decreases consumer surplus. 
The total consumer surplus in the economy is

$$
\int_{0}^{1} b(c) \pi(\eta(c))-w(\eta(c)) d c=\int_{\hat{\eta}}^{\infty} b(c(\eta)) \pi(\eta)-w(\eta) d c(\eta) .
$$

Before we formally prove disclosure always decreases this surplus, it is worth first understanding the intuition. What the above reasoning ignores is how equilibrium prices react to the disclosure of information. To make things transparent, assume that all consumers have the same willingness to pay, $b(c)=1$, and the opportunity cost is zero, $A=0$. Take an equilibrium that features charlatans. Now assume a government entity exists that can perfectly identify who the charlatans are. If such a government entity wished to maximize consumer surplus, would it reveal this information? To see why the answer is no, note that if the government revealed the information, the equilibrium would collapse to the equilibrium that features no charlatans. As we have already seen, in that equilibrium the wage is $b(1-\lambda)=1$, implying that all consumers earn zero surplus:

$$
b(c)-w=b(c)-b(1-\lambda)=0 .
$$

However, if the government does not reveal the information, consumer surplus is positive. To see why, notice from (8) that

$$
\begin{aligned}
w(\eta) & =\int_{\bar{\eta}}^{\eta} b(c(v)) \pi^{\prime}(v) d v-A \\
& =\int_{\bar{\eta}}^{\eta} \pi^{\prime}(v) d v \\
& =\pi(\eta)-\pi(\bar{\eta}) .
\end{aligned}
$$

Thus consumer c's surplus is

$$
\begin{aligned}
\pi(\eta(c)) b(c)-w(\eta(c)) & =\pi(\eta(c))-\pi(\eta(c))+\pi(\bar{\eta}) \\
& =\pi(\bar{\eta})>0
\end{aligned}
$$

implying that the total consumer surplus in the economy is positive. Consequently, revealing who the charlatans are makes consumers worse off.

In this example, all consumers are made worse off by the disclosure of information. However, when consumers do not value the service equally, it is possible that the revelation of information can make some consumers better off. Even so, if the skill is in short enough 
supply, the following proposition (proved in Appendix C) proves that if a market exists in which the service is provided, any disclosure of information lowers total consumer surplus:

Proposition 1 There exists a $\hat{\lambda}>0$ such that in an equilibrium of any economy in which $\lambda<\hat{\lambda}$, consumer surplus is strictly increasing in $\sigma$.

What this proposition implies is that, subject to one exception, a government that wishes to maximize consumer surplus and has better information than consumers, will never choose to reveal that information. The sole exception occurs if no equilibrium exists, and by revealing some information the government can restore the equilibrium. Of course, even in this case, the government would choose to reveal only enough information to just restore the equilibrium, and no more. Either way, full disclosure is not optimal.

As we have already said, the reason that the usual "disclosure allows consumers to make better choices" argument fails to imply that it also benefits consumers, is that it ignores the equilibrium price response. To see why explicitly, recall, from (10) how wages are determined:

$$
w(\eta)=b(c(\eta)) \pi(\eta)-\lambda \int_{\bar{\eta}}^{\eta} b^{\prime}(c(v)) f_{s}(v) d v-A .
$$

implying that consumer surplus in this case is

$$
b(c(\eta)) \pi(\eta)-w(\eta)=\lambda \int_{\bar{\eta}}^{\eta} b^{\prime}(c(v)) f_{s}(v) d v+A .
$$

That is, because the first term in the wage function is the expected benefit (in expectation, consumers are not fooled), in every equilibrium they get more than what they pay for. Thus, the disclosure of information does not help consumers make better choices. Instead what it does is reduce competition, which reduces the magnitude of the next term in the equilibrium wage function, thus reducing consumer surplus. Examining this term is revealing. Because $\bar{\eta}$ is decreasing in $\sigma$, when information is revealed $\bar{\eta}$ increases, which removes skilled producers from the market, thus decreasing competition. There is, however, a possible countervailing effect because of the dependance of $c(\eta)$ and $f_{s}(\eta)$ on $\sigma$. For some consumers, in particular, those consumers with very high willingness to pay, this dependence can dominate the effect of the reduced competition. Intuitively, when there is a lot of noise in the signal, it is difficult to differentiate producers. Consumers with a high willingness to pay have to compete with other high willingness-to-pay consumers for the very few producers with high signals. Thus, they get little surplus. However, when there is less noise, these consumers can better identify the skilled producers. That increases 
supply of skilled producers who can be identified, and thus increases consumer surplus. While this is true for some consumers, for most consumers, surplus is reduced because they essentially pay nothing and get nothing. What the proposition proves is that the aggregate effect leaves consumers as a whole worse off.

To assess the magnitude of the reduction in total consumer surplus, we can compare an equilibrium with and without uncertainty under a range of different parameters. To do this, assume that $b(x)=x$. As we have already seen, the equilibrium wage under perfect information is $b(1-\lambda)=1-\lambda$. Total consumer surplus in an economy with perfect information is therefore

$$
\int_{0}^{1} b(c) \pi(\eta(c))-w(\eta(c)) d c=\int_{1-\lambda}^{1} c-(1-\lambda) d c=\frac{\lambda^{2}}{2} .
$$

In an economy with uncertainty, consumer surplus is given by

$$
\int_{\hat{\eta}}^{\infty} b(c(\eta)) \pi(\eta)-w(\eta) d c(\eta)=\lambda \int_{\bar{\eta}}^{\infty} \int_{\bar{\eta}}^{\eta} f_{s}(v) d v d c(\eta)+A .
$$

Thus, the difference in consumer surplus between the two equilibria, expressed as a fraction of consumer surplus in the economy with perfect information, is

$$
\frac{\lambda \int_{\bar{\eta}}^{\infty} \int_{\bar{\eta}}^{\eta} f_{s}(v) d v d c(\eta)+A-\frac{\lambda^{2}}{2}}{\frac{\lambda^{2}}{2}}=\frac{2}{\lambda} \int_{\bar{\eta}}^{\infty} \int_{\bar{\eta}}^{\eta} f_{s}(v) d v d c(\eta)+\frac{2 A}{\lambda^{2}}-1
$$

Figure 1 plots this difference as a percentage of the surplus in the full information equilibrium, over a range of parameter values. The effect is large, even when $\lambda$ is as high at 0.2 and $A=0$ - when the signal noise is large, the percentage difference is well over $100 \%$. For small values of $\lambda$ the percentage difference explodes. As the signal noise decreases and the equilibrium converges to the perfect information equilibrium, the percentage difference shrinks to zero.

Charlatans impose costs because they are paid positive wages yet produce nothing. Because, in expectation, consumers do not bear these costs, they can only borne by skilled producers. Consequently, skilled producers benefit from disclosure. Disclosure reduces the cost imposed by charlatans and consequently increases skilled workers' expected wages. To formally demonstrate this, first note that the (ex-ante) expected wage of a skilled 

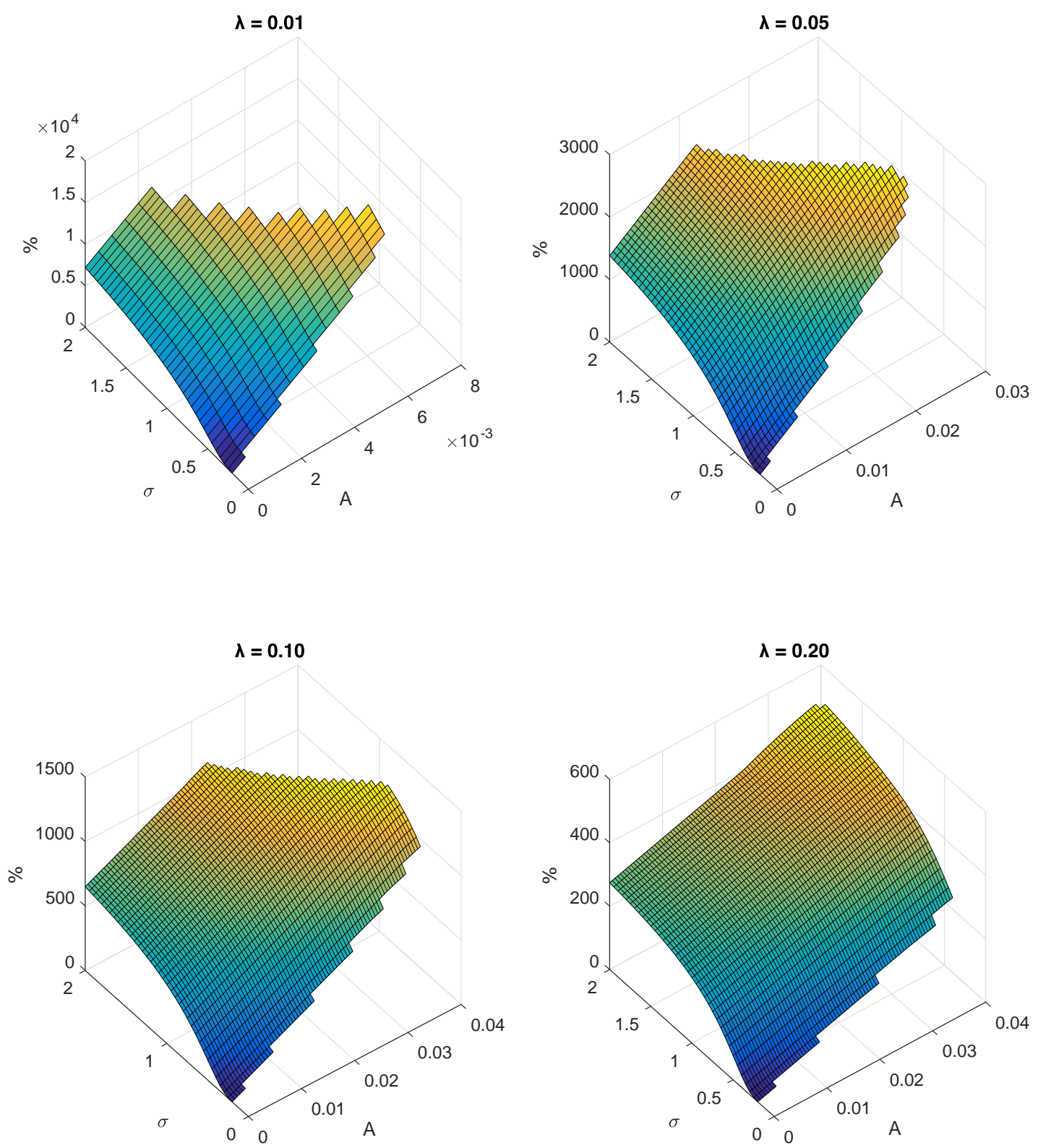

Figure 1: Percent Difference in Consumer Surplus: Each plot is (13), the difference between consumer surplus in an equilibrium with uncertainty and a economy with perfect information as fraction of the surplus in the full information economy, quoted in percent, as a function of informativeness of the signal $(0.2 \leq \sigma \leq 2)$ and the opportunity cost $(0 \leq A \leq 0.04)$ in the economy with uncertainty. Each plot varies the measure of skilled producers, that is, $\lambda=0.01,0.05,0.1,0.2$. Whitespace in the plots corresponds to regions in which equilibria do not exist in the case with uncertainty. 
agent is

$$
\int_{\hat{\eta}}^{\infty} w(\eta) f_{s}(\eta) d \eta-A F_{s}(\hat{\eta})=\int_{\hat{\eta}}^{\infty} \int_{\bar{\eta}}^{\eta} b(c(v)) \pi^{\prime}(v) f_{s}(\eta) d v d \eta-A
$$

The next proposition (proved in Appendix D) shows that the expected wage of a skilled worker is increased when information is disclosed:

Proposition 2 There exists a $\hat{\lambda}>0$ such that in an equilibrium of any economy in which $\lambda<\hat{\lambda}$, the expected wage of skilled workers is strictly decreasing in $\sigma$.

The main implication of this proposition is that it is always in the interest of skilled workers to credibly disclose information. As a consequence, one would expect skilled workers to form trade groups to credibly reveal quality. This insight likely explains the source of the data used in Egan et al. (2016) — data made publicly available by the trade group that represents financial analysts, FINRA. Since skilled workers can always choose to disclose information, the only reason why uncertainty about quality can remain, is if either no mechanism exists that allows skilled workers to credibly disclose, or if it is too costly for skilled workers to implement such a mechanism.

One last question one can ask is how the disclosure of information affects overall welfare within the high skill sector. Overall welfare might be affected in two ways. First, information disclosure will affect the total supply of skill in the sector because it affects $\bar{\eta}$, which in turn affects the supply of skilled workers. Second, workers who choose to obtain a signal but then choose not to provide the service suffer an opportunity cost. Thus overall welfare is total supply of skill minus the overall opportunity cost incurred:

$$
\left(1-F_{s}(\bar{\eta})\right)-A\left(F_{s}(\bar{\eta})+F_{u}(\bar{\eta})\right)
$$

The following proposition shows that the disclosure of information increases overall welfare.

Proposition 3 There exists a $\hat{\lambda}>0$ such that in an equilibrium of any economy in which $\lambda<\hat{\lambda}$, overall welfare in the sector is strictly decreasing in $\sigma$.

The intuition behind this proposition is straightforward. By disclosing information skilled can be better measured, which means that in equilibrium less skilled workers fail to make the market imposed minimum standard $\bar{\eta}$. That implies the supply of skill is increased in equilibrium. Because consumer surplus decreases, what this means is that 
producers capture all marginal welfare gains. In that sense producer interests are aligned with overall welfare in the sector.

When skilled workers do not have the means to disclose information about quality, the equilibrium will contain charlatans (provided that it exists). So a natural question to ask is whether in this setting exogenously imposed standards can be beneficial. The literature that studies this question does so in the context of certification and licensing. It has generally favored certification because certification does not constrain consumers. What the first proposition implies is that certification is never an optimal strategy to increase consumer surplus. Certification reduces consumer surplus because its only role is to disclose information. Proposition 2 implies that the release of information that accompanies certification benefits skilled producers. If, after certification requirements have been implemented, residual uncertainty remains, what we will show in the next section is that implementing a licensing requirement further benefits skilled workers at the cost of consumers. However, since licensing and certification convey the same information, what that means is that skilled workers always prefer licensing to certification. That is, in this setting certification is never the preferred outcome for either producers or consumers.

\section{Regulation of Standards}

A defining characteristic of the equilibrium in the previous section is that it always features charlatans. This immediately raises the question of whether a minimum standard might increase welfare by limiting the entry of charlatans. The literature that has studied standards has focused on two methods to implement a standard. As we pointed out in the introduction, under the certification standard, a trade group certifies that a producer has met its requirements, but producers that have not met the requirements are still allowed to practice. Under the licensing standard, producers who do not meet the standard are not allowed to work. Thus the role of certification is only the communication of information, which is what we studied in the previous section. But the question remains, how does a licensing requirement affect the utility of consumers and producers?

To investigate the effect of licensing, we introduce the requirement that a producer may only produce if her signal exceeds a lower bound $\hat{\eta}$. Obviously, in any equilibrium without a minimum standard, introducing a minimum standard below $\bar{\eta}$ has no effect. So we only consider the case where $\hat{\eta} \geq \bar{\eta}$.

In Appendix B we derive the conditions for existence of an equilibrium with a minimum 
standard. Appendix B contains two propositions. Proposition 8 shows that for any minimum standard $\hat{\eta} \geq \Delta$, where $\Delta$ solves $\lambda F_{s}(\Delta)+F_{u}(\Delta)=\lambda$ an equilibrium always exists. The intuition is similar to the derivation of the equilibrium without a standard. The main difference is that the minimum standard is now exogenously set rather than determined in equilibrium. Because the standard is higher than $\bar{\eta}=\Delta$, not all consumers can consume. The constant of integration in the wage function is therefore set such that the marginal consumer is indifferent between consuming or not, rather than before where it was set to ensure that the marginal producer was indifferent between producing or not. Proposition 9 derives the existence condition when $\hat{\eta}<\Delta$. In this case, all consumers consume because the standard is below $\Delta$ and we are in the region where unskilled producers are indifferent to becoming charlatans. That indifference condition now determines the constant of integration in the wage function.

In the $\hat{\eta} \geq \Delta$ case there are three possibilities depending on the initial parameters: All unskilled agents choose to become charlatans $(y=1)$, no unskilled agent chooses to become a charlatan $(y=0)$ or a fraction of unskilled agents chooses to become charlatans $(0<y<1)$. In all cases $c(\eta)$ and $w(\eta)$ are given by:

$$
c(\eta)=1-\lambda\left(1-F_{s}(\eta)\right)-y\left(1-F_{u}(\eta)\right)
$$

and

$$
w(\eta)= \begin{cases}b(c(\eta)) \pi(\eta)-\lambda \int_{\hat{\eta}}^{\eta} b^{\prime}(c(v)) f_{s}(v) d v & \text { if } y>0 \\ b(c(\hat{\eta})) & \text { if } y=0\end{cases}
$$

where we have rewritten the expression for wages in Proposition 8 by integrating by parts and substituting.

There are two crucial differences between this equilibrium and the equilibrium without a minimum standard. First, a no charlatan equilibrium exists - it is possible to set the standard high enough so that equilibrium will feature no charlatan entry. Second, so long as the opportunity cost is positive, when a standard is introduced, wages strictly increase. The reason wages are higher is that they no longer need to perform the role of keeping charlatans out. The minimum signal has that role. What that implies is that when $y>0$, wages are equal to the expected benefit minus a term that only takes into account the degree of competition from other skilled producers currently in the market, but not potential producers (currently not in the market), as in the case without a minimum standard. Notice that when the minimum standard is set so high that no unskilled 
worker chooses to be a charlatan, then all producers earn the same wage (because they are identical). Competition between them lowers the wage to the benefit conferred by the producer with the lowest allowable signal.

The fact that, by imposing a minimum standard, it is possible to have equilibria that contain no charlatans raises the question of whether such equilibria are desirable. To investigate this question we again consider the effect from the perspective of consumers and skilled producers. We will assume that one of two entities can impose a minimum signal, $\hat{\eta}$, that must be attained for a producer to be allowed to produce. The first entity is an organization like a government that represents the interests of consumers. This entity's objective is to pick a minimum standard that maximizes consumer surplus. The second entity is a trade group that represents skilled agents. This trade group maximizes the ex-ante expected wage of skilled agents.

We begin by deriving the government's optimal policy. The next proposition (with proof in Appendix F) proves that a government would never introduce a minimum standard into an equilibrium that does not have a minimum standard.

Proposition 4 There exists a $\hat{\lambda}>0$ such that in an equilibrium of any economy in which $\lambda<\hat{\lambda}$, introducing a minimum standard reduces consumer surplus.

The main implication of this proposition is that, subject to one exception, a government maximizing the interests of consumers within the industry would never impose a licensing requirement. The one exception is when, without a minimum standard, the market breaks down and there is no equilibrium. Because there is always a minimum standard such that an equilibrium exists that features skilled workers, by imposing a standard, the government can create a market that otherwise would not exist. Because consumer surplus is zero without the market and positive with the market, introducing a minimum standard will increase surplus in this case. But in this case, any standard that is introduced will be the minimum standard that is required to restore the equilibrium. Any higher standard will reduce consumer surplus.

The explanation for this seemingly nonintuitive result is the same as the explanation for why divulging information is bad for consumers. Wages in the equilibrium without a standard fully take into account the probability that a producer is skilled. Put differently, nobody is fooled in the economy, consumers in expectation get what they pay for. So the only effect of introducing a minimum standard is to reduce the entry of producers, thereby reducing competition, and thus increasing wages. There are two independent forces that 
drive up wages. First, as we have already pointed out, wages are not reduced by the term required to keep out charlatans (that is, A). In addition, because the equilibrium features fewer producers, the term that accounts for the competition between producers is also lower. Because wages are higher, consumer surplus is lower.

One might wonder how any exogenously imposed minimum standard in a market for professional services in a field like medicine (which has high costs for mistakes) always results in a welfare loss. Surely, a minimum standard that eliminates the worst mistakes would be desirable? But the point to realize is that labor markets without exogenous minimum standards have market imposed standards. That is, wages adjust so that no producer with a signal below $\bar{\eta}$ chooses to produce. Those that do clear the bar by a small margin, earn very low wages. So, for a profession like medicine, most consumers highly value successful treatment and so without an exogenously imposed minimum standard receiving quality treatment has a high price. The insight is that with an exogenously imposed minimum standard it is even more costly. By allowing the market, rather than the government, to sort out skilled producers from charlatans, consumers benefit.

Given that government imposed licensing requirements are ubiquitous in highly skilled professions, a natural question to ask is why they exist in light of the above proposition. We believe the answer is that skilled producers benefit from licensing, and so when trade groups are powerful enough, they are able to lobby government legislatures to act in their interest rather than consumers'. The next proposition (with proof in Appendix G) shows that a trade group will always favor introducing a licensing requirement into an equilibrium that does not have such a requirement (including economies that already have certification requirements).

Proposition 5 There exists a $\hat{\lambda}>0$ such that in an equilibrium of any economy in which $\lambda<\hat{\lambda}$, introducing a minimum standard always strictly increases the expected wage of skilled workers.

The proof of this proposition relies on two insights. First, because wages reflect the information in the signal, consumers do not bear the cost of charlatans. Instead, the cost is born by skilled workers. Therefore, anything that reduces the number of charlatans benefits skilled workers. A minimum standard does this, but with a cost — some skilled workers will be caught by the standard and will be unable to work. But because $F_{s}$ stochastically dominates $F_{u}$, the minimum standard hits charlatans harder then skilled workers. In cases where the cost to skilled workers is small, it is optimal to set the standard high enough so no unskilled workers choose to be charlatans. 
Second, imposing a minimum standard fundamentally changes the terms of trade. Without a minimum standard, wages have to adjust to limit charlatan entry. When there is a minimum standard, wages adjust to limit consumers, implying that wages are no

longer needed to be reduced by the opportunity cost of providing the service.

How do standards affect overall welfare within the high skill sector? The following proposition shows that introducing a standard decreases welfare.

Proposition 6 Any minimum standard that exceeds $\bar{\eta}$ strictly decreases overall welfare in the sector.

The proof of this proposition is straightforward. Increasing the minimum standard decreases the supply of skill in the economy and increases the number of producers who have to bear the opportunity cost. In this case consumers bear all the welfare losses so consumer interests are aligned with overall welfare.

If one takes the perspective that the licensing standard we see reflects the interests of trade groups, then the wide variation in standards across professions documented in Section 1 must reflect cross sectional differences in the tradeoffs faced by trade groups in these professions. With that in mind, in the next section we investigate how cross sectional differences in professions affect the licensing standards that trade groups favor.

\section{$5 \quad$ Factors Driving Variation in Licensing Standards}

In this section we assume that the trade group picks the standard that maximizes the expected wage of skilled workers. For simplicity, we assume that $b(x)=x$, that is, consumer willingness to pay for the service linearly increases. Under this assumption, we solve for the optimal standard given the fraction of skilled agents, $\lambda$, the informativeness of the signal, $\sigma$, and the opportunity cost, $A$.

\subsection{Charlatans}

As we have already pointed out, when the trade group sets standards, it does so by trading off the benefit of reducing the number of charlatans against the cost of a standard that catches some unlucky skilled workers. So the question is, when is it optimal for the trade group to set a standard so high that it discourages all charlatan entry? Notice that when $A=0$, there is no standard that will accomplish this goal. Because unskilled workers face no cost to become charlatans, it is impossible to discourage them. At the other extreme, 


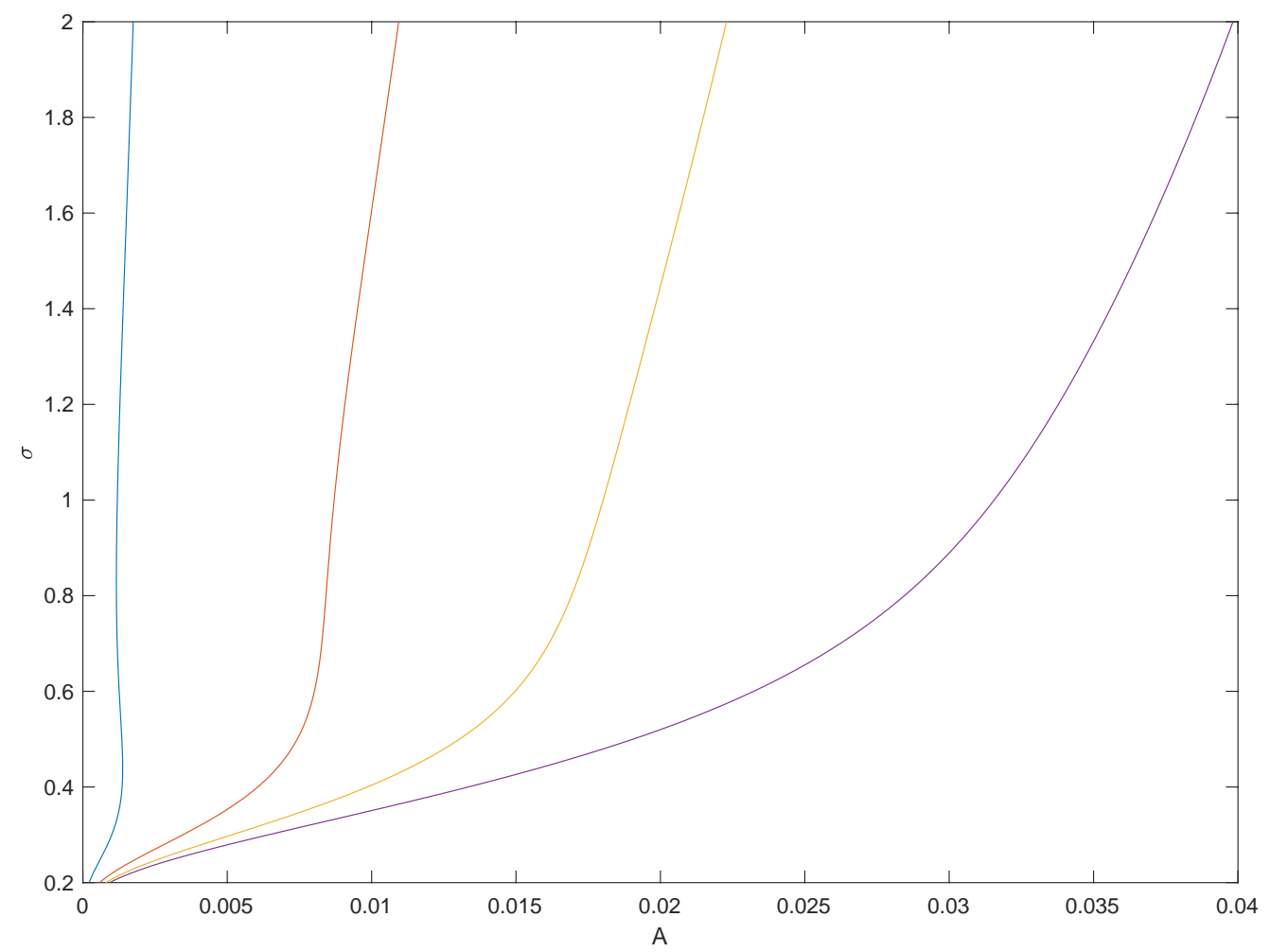

Figure 2: Charlatans in Equilibrium: Each curve plots the boundary between equilibria that feature charlatans and equilibria that do not when a trade group picks the optimal minimum standard as a function of the informativeness of the signal $(\sigma)$ and the opportunity cost $(A)$. Points to the left of the curve are equilibria that feature charlatans. Points to the right are equilibria that do not feature charlatans. The blue, red, yellow and purple lines represent equilibria with $\lambda=0.01,0.05,0.10$ and 0.20 .

when $A$ is very large and $\sigma$ is low, it will be possible to pick a standard that is low enough so most skilled workers are able to meet the standard, but most unskilled workers will not. Given the high opportunity cost, the unskilled workers will find it suboptimal to become charlatans and the equilibrium will feature only skilled workers. Consequently, when the trade group is allowed to pick a standard that maximizes the expected wages of skilled workers, there will exist a set of parameters under which equilibria will feature charlatans and a complimentary set where equilibria will not feature charlatans. Figure 2 plots these two sets.

Each curve in Figure 2 plots the boundary between these two sets for four different values of $\lambda$, the amount of skilled workers. Points to the left of the curve are equilibria 

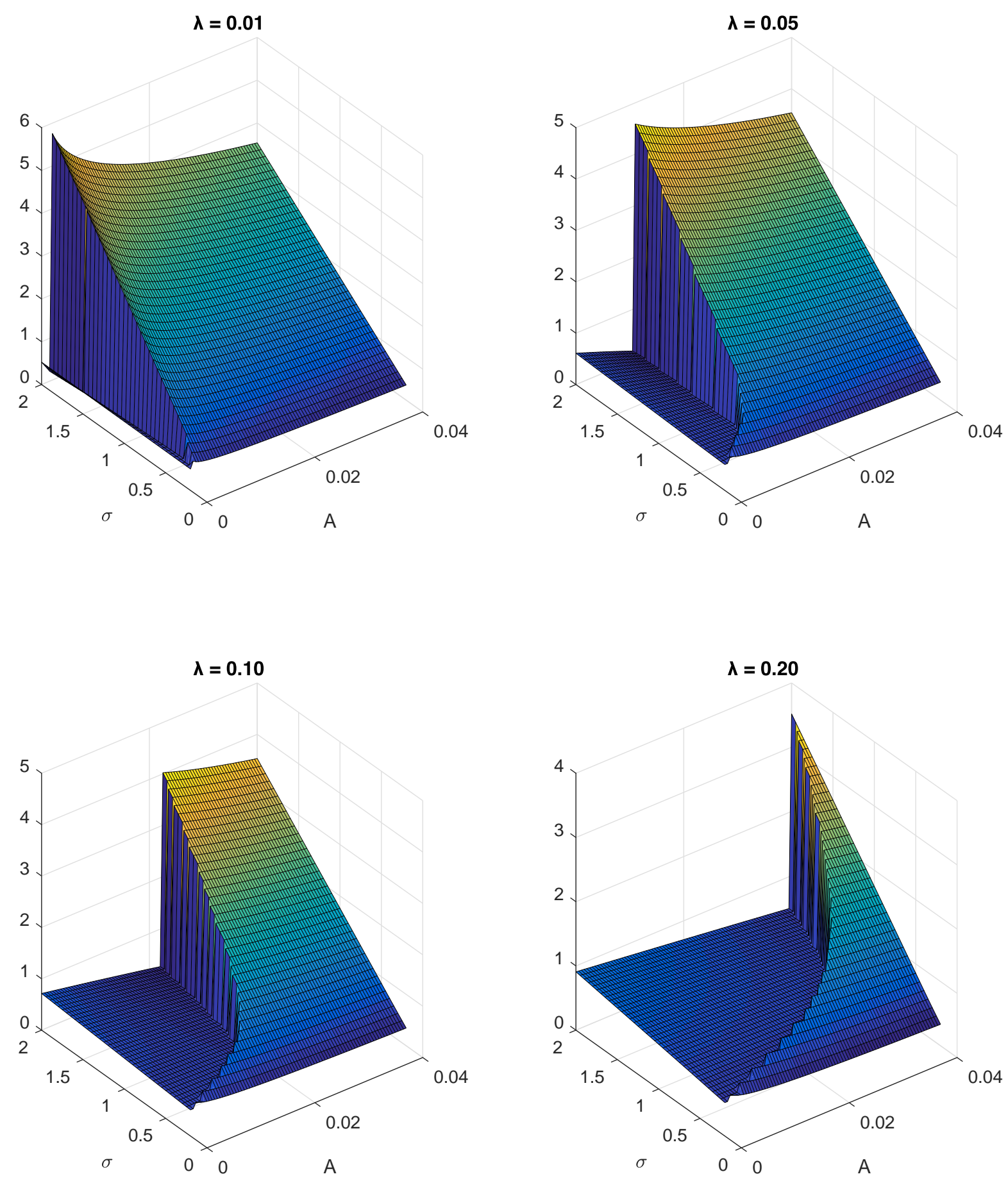

Figure 3: Minimum Standard Imposed by the Trade Group: Each plot is the minimum standard imposed by the trade group as a function of the informativeness of the signal $(\sigma)$ and the opportunity cost $(A)$. The plots vary the supply of skill in the economy, that is, starting with the top left plot and moving clockwise $\lambda=0.01,0.05,0.20$ and 0.10 . The "cliff" in the plots corresponds to the demarcation (plotted in Figure 2) between equilibria that feature charlatans and ones that do not. 
that feature charlatans. Points to the right are equilibria that do not feature charlatans. As we already pointed out, when $A=0$, there is no cost to becoming a charlatan, so all equilibria feature charlatans. Counterintuitively, when $\lambda$ is small, it does not take much of an increase in $A$ to discourage charlatan entry. When skill is in short supply one would expect very high wages, and so at first glance one might suppose that it would be very difficult to keep charlatans out. But, in fact, the equilibrium effect goes the other way. Because the skill is in such short supply, without a minimum standard the equilibrium is dominated by charlatans and thus features very low wages. Setting a standard high enough to keep charlatans out is, in fact, not that costly in this case, because the skilled workers that are denied work would have earned low wages anyway in the equilibrium with no minimum standard. So, from the trade group's perspective, the optimal policy is to set a very high standard (see, Figure 3, top left) which implies that even for a small opportunity cost, the no charlatan equilibrium (albeit with very small supply of skilled workers) prevails.

When the number of skilled workers is larger (purple curve in Figure 2), the difference in wages between the charlatan and no-charlatan equilibrium is lower, so the cost to skilled workers of setting a high standard is high. Consequently, it is optimal for the trade group to set a lower standard (see, Figure 3, bottom right), which implies that a higher $A$ is required to produce the no-charlatan equilibrium.

The effect of the informativeness of the signal is intuitive and easy to understand. As the signal is less informative, it is easier for charlatans to pose as skilled workers. Consequently, a very high standard is required to keep them out. When the supply of skill is higher it becomes too costly to impose such a high standard, so in this case the trade group favors a lower standard that results in an equilibrium that features charlatans. Figure 2 demonstrates this tradeoff. When the number of skilled workers is low, most equilibria have high standards (see Figure 3, top left) and no charlatans. But when the supply of skill is higher the trade group opts for much lower standards (see Figure 3, bottom right), and accepts the presence of charlatans.

These results are informative about the descriptive data presented in Section 1. Note that for professions with skill in short supply and high opportunity costs, the equilibrium does not feature charlatans (blue and red curves in Figure 2). To get that equilibrium, the trade group has to set very high standards (Figure 3, upper plots). Both facts are consistent with the evidence in the medical profession. On the other hand, when the opportunity cost is low, the equilibrium features charlatans and low standards, which is 
consistent with the finance industry. When the supply of skill is higher, the quality of the information matters more. When there is high uncertainty, charlatan equilibria dominate (purple curve in Figure 2), which is at least consistent with the short training period and low standards in the real estate industry, suggesting that the industry has a high fraction of charlatans. Finally, accountants have higher opportunity costs, but high standards, suggesting that the profession is likely to feature fewer charlatans.

\subsection{Consumer Surplus}

When the trade group chooses to set a high standard and the result is an equilibrium that does not feature charlatans, the lack of competition translates into very low consumer surplus, see Figure 4. Competition is reduced for two reasons. First, there are no charlatans. But, in addition, because high standards are required, the standard reduces the supply of skilled agents, further reducing competition. Second, consumers are also hurt because these high standards reduce the overall supply of skill that is available for consumption. This is particularly true when the signal is uninformative because very high standards are needed to keep charlatans out. When the signal is informative, standards can be set that simultaneously discourage charlatans without significantly reducing the supply of skilled workers. Thus, for low $\sigma$, consumer surplus is higher. The effect on the supply of skilled workers of the minimum standard can be seen in Figure 5. When the standard is set very high and the signal is not very informative, the supply of skilled workers who make the standard is small, leading to the low consumer surplus results in Figure 4.

\subsection{Wages}

Figure 6 plots the expected wage of skilled workers. That is, the expected value of being hired at the equilibrium wage and not making the standard (and thus earning the reservation wage). What the plots make clear is that the first order determinant of the expected wage is the informativeness of the signal. As the signal becomes less informative, the presence of charlatans decreases expected wages. This is true even in the equilibria that feature no charlatans. In that case, the minimum standard is set so high that most skilled workers cannot make the standard and so earn the reservation wage. That is, either the equilibrium features charlatans and the inability of consumers to separate them lowers wages, or it does not feature charlatans, but the cost to keep them out implies that many skilled workers are denied work and thus earn the reservation wage. 

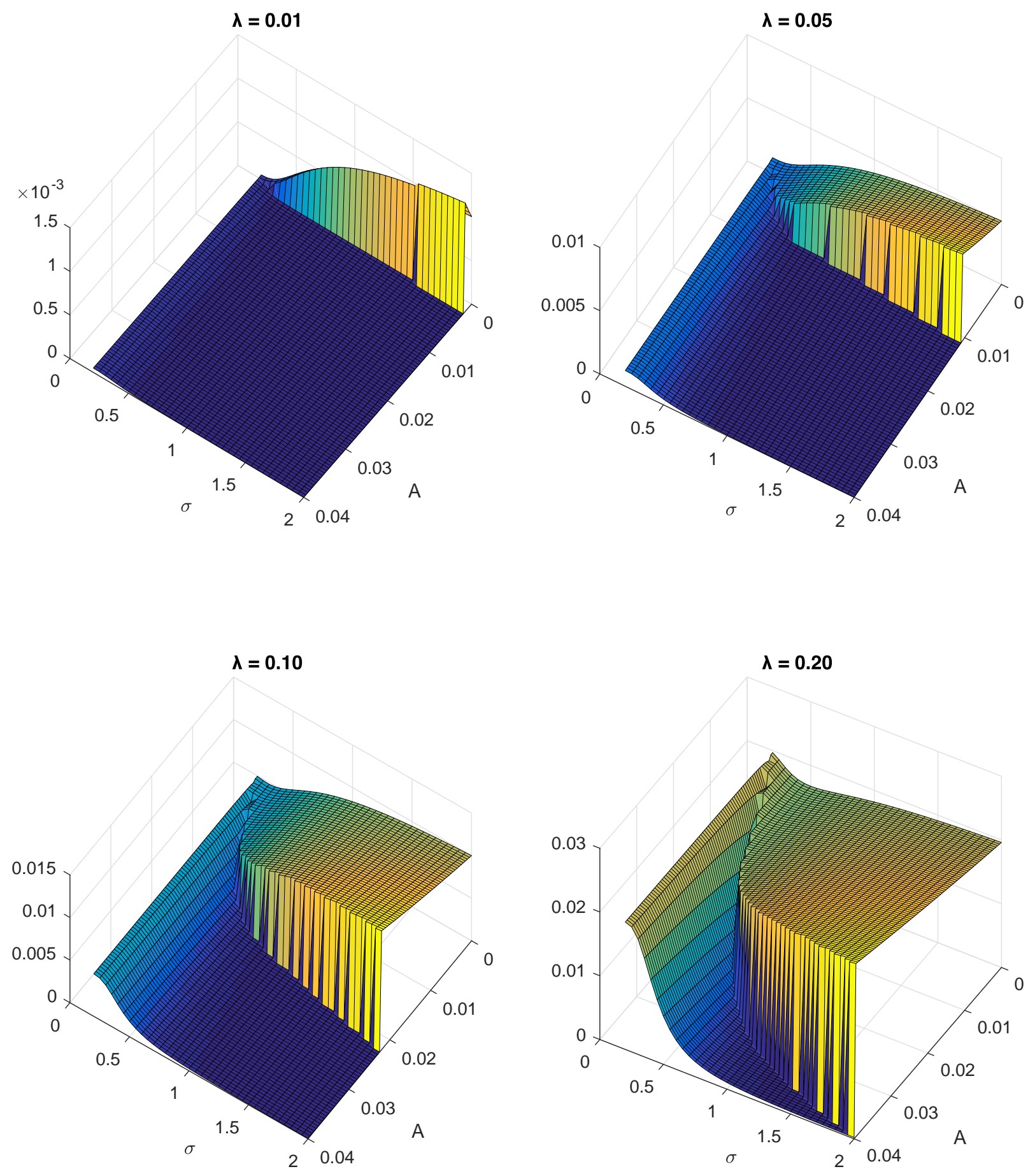

Figure 4: Consumer Surplus: Each plot is the equilibrium level of consumer surplus given a minimum standard imposed by the trade group as a function of the informativeness of the signal $(\sigma)$ and the opportunity cost $(A)$. The plots vary the supply of skill in the economy, that is, starting with the top left plot and moving clockwise $\lambda=0.01,0.05,0.20$ and 0.10 . 

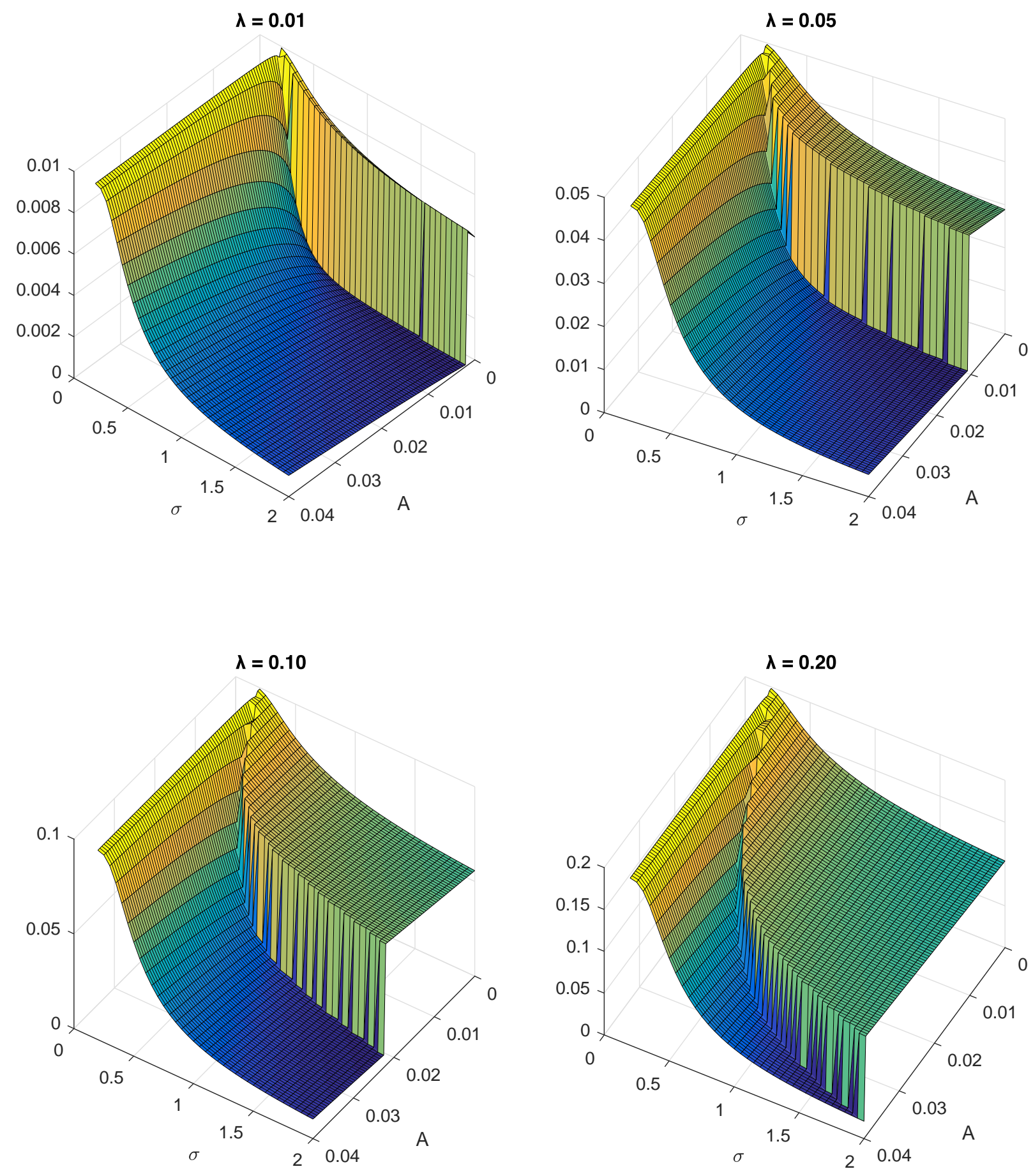

Figure 5: Equilibrium Supply of Skilled Agents: Each plot is the equilibrium supply of skilled agents (that is, the supply once the standard is imposed) as a function of the informativeness of the signal $(\sigma)$ and the opportunity cost $(A)$. The plots vary the supply of skill in the economy, that is, starting with the top left plot and moving clockwise $\lambda=0.01$, $0.05,0.20$ and 0.10 . 

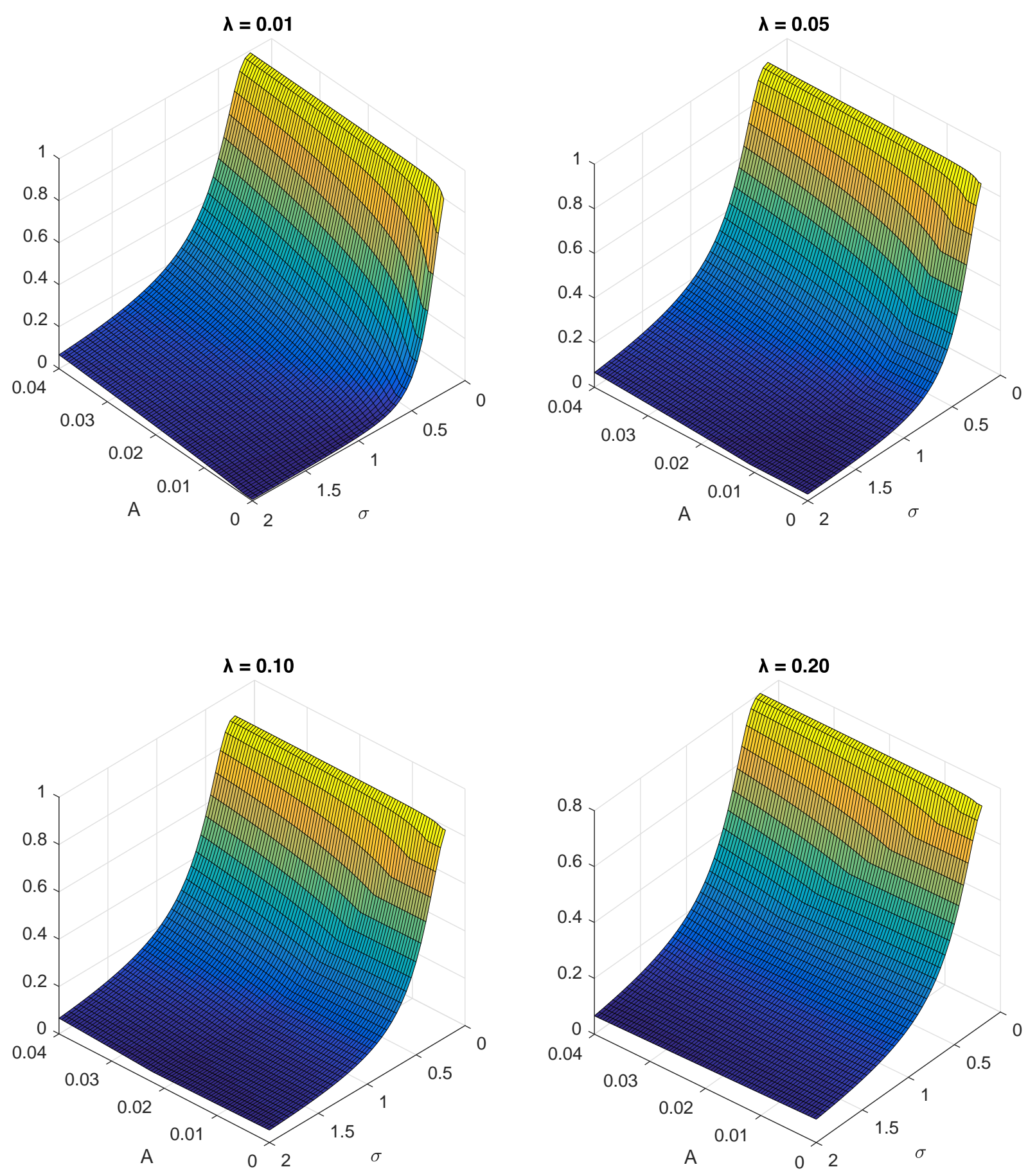

Figure 6: Expected Wage of Skilled Agents: Each plot is the expected wage of skilled agents when the minimum standard is imposed by the trade group as a function of the informativeness of the signal $(\sigma)$ and the opportunity cost $(A)$. The plots vary the supply of skill in the economy, that is, starting with the top left plot and moving clockwise $\lambda=0.01$, $0.05,0.20$ and 0.10 . 
In contrast, actual wages, that is the wages of agents who make the standard look quite different. As Figure 7 shows, in equilibria that do not feature charlatans, the average wage is very close to 1 , implying that the skilled workers who do make the standard extract almost all the rents. This is particularly true in cases with low $\lambda$ and low $A$, where the minimum standard is very high. In contrast, when the equilibrium has charlatans, wages are low, reflecting the inability of consumers to separate skilled workers from charlatans and the fact that consumers obtain some of the rents.

The last figure, Figure 8, plots the cross-sectional standard deviation of wages. Clearly, in equilibria with no charlatans, since all agents earn the same wage, the standard deviation is zero. What is interesting is that for most parameter values even equilibria that feature charlatans have a very low cross sectional standard deviation in wages. The reason is that when the signal is uninformative, wages do not vary much. The only cases where wages feature large cross sectional variation, is equilibria where the opportunity cost is low enough so that even when the signal is informative, the equilibrium features charlatans. As the figures make clear, this case is most common when $\lambda$ is high.

\section{Discussion}

The explanation we explore in this paper for the explosive growth in the last 50 years of licensing requirements is that trade groups are successful in lobbying governments to act in their interests. If this is not the explanation, then one of the assumptions of our model that leads to this conclusion has to be violated. In this section we identify the assumptions that we believe are most critical for our results and discuss the likelihood that they do not hold in reality.

Our model provides benchmark results for a labor market where skill is in very short supply (and high demand). The extent to which our model applies to professions where the skill is in larger supply depends on the elasticity of the supply of skilled professionals to wages. As we have already mentioned, in labor markets that require an extensive trading period, this elasticity is likely to be low in the short term. For such labor markets, our model applies in the short term.

In the long term, the spurring of skilled entry into such professions could make consumers potentially better off (Leland (1979)). If indeed this is the correct explanation for the increased trend in regulation, we should see cross-sectional variation across pro-

fessions in the amount of regulation: professions, like finance and medicine, with skills 

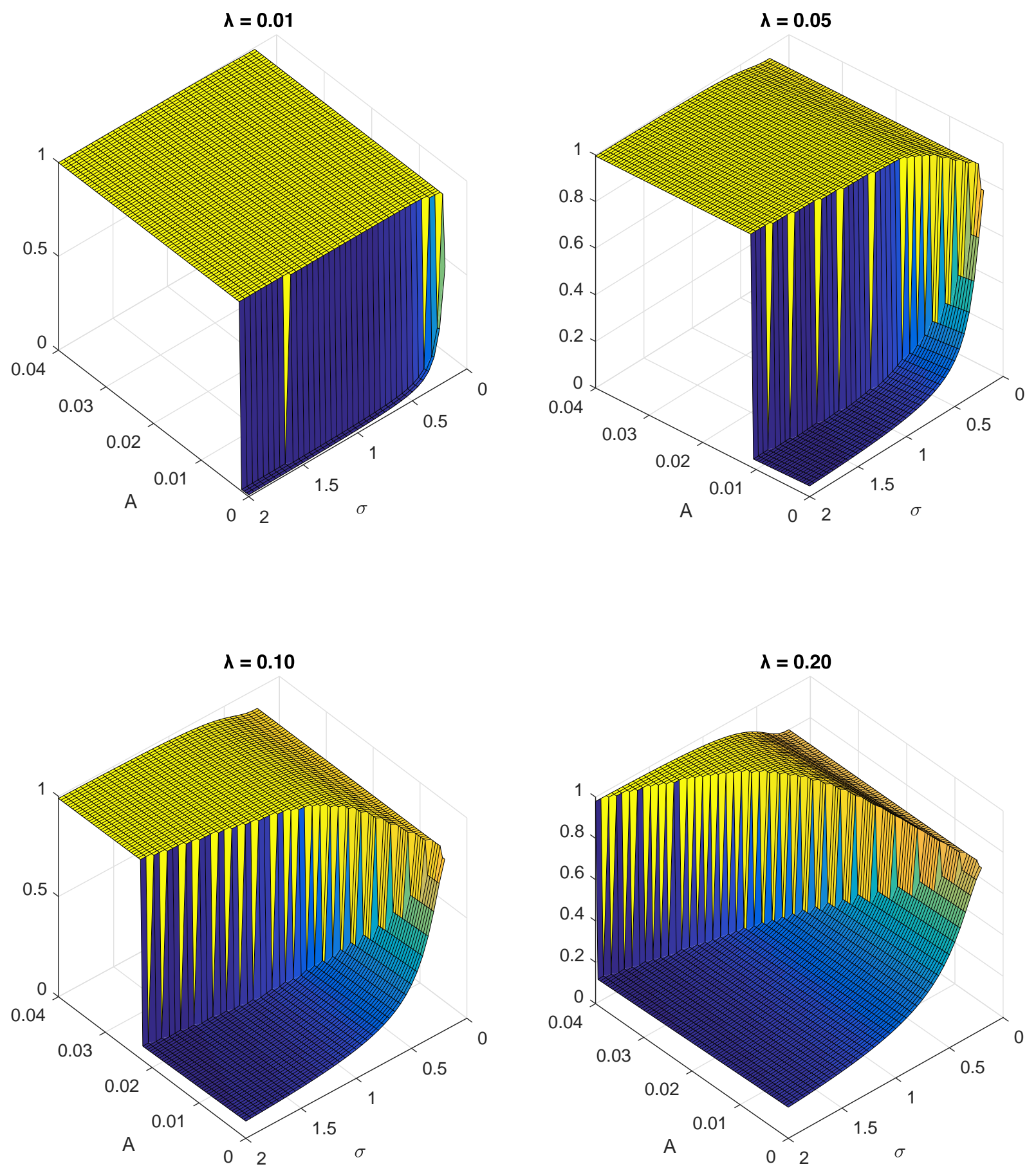

Figure 7: Realized Mean Wages: Each plot is the mean realized wage when the minimum standard imposed by the trade group as a function of the informativeness of the signal $(\sigma)$ and the opportunity cost $(A)$. The plots vary the supply of skill in the economy, that is, starting with the top left plot and moving clockwise $\lambda=0.01,0.05,0.20$ and 0.10 . 

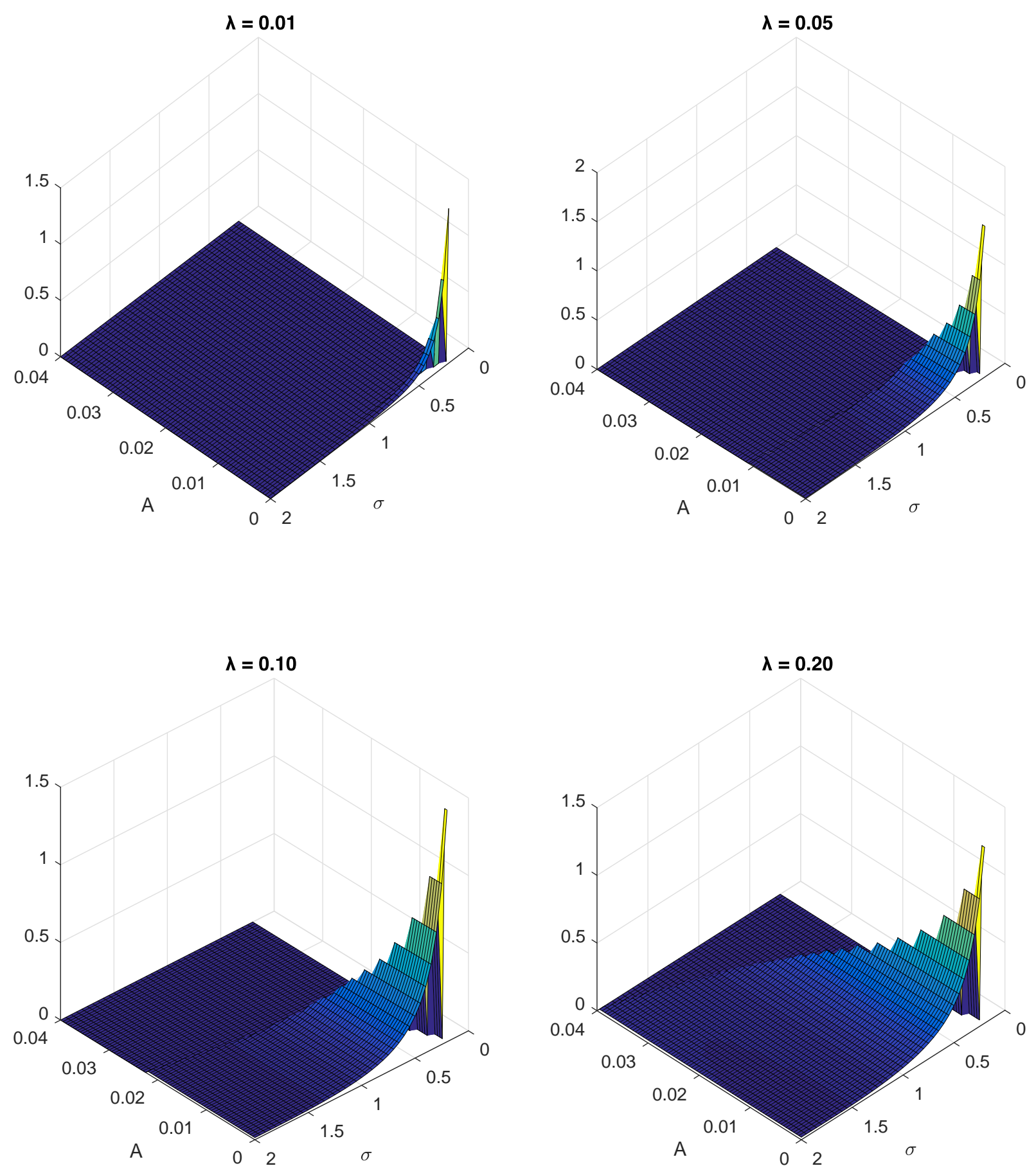

Figure 8: Realized Standard Deviation of Wages: Each plot is the equilibrium standard deviation of realized wages when the minimum standard imposed by the trade group as a function of the informativeness of the signal $(\sigma)$ and the opportunity cost $(A)$. The plots vary the supply of skill in the economy, that is, starting with the top left plot and moving clockwise $\lambda=0.01,0.05,0.20$ and 0.10 . 
in very short supply should have little regulation and professions with a skill in higher supply like real estate agents should be more heavily regulated. Anecdotally, at least, the evidence appears go the other way - medical doctors are clearly more regulated than real estate agents. Future empirical work could focus on clarifying these relationships.

A second assumption in our model is that all participants are fully rational. In particular, consumers appropriately take into account the probability of dealing with a charlatan. If, instead, prices of services do not accurately reflect that probability, for example, because consumers are naive, government intervention to protect consumers from themselves, could be beneficial. Effective government policy in such cases requires that the government be more rational than consumers and well informed about consumer preferences.

A third assumption in our model is that the willingness to pay function is not a function of the level of uncertainty $\sigma$. That is, consumers are effectively risk neutral to this uncertainty. If they are averse to this uncertainty, then by disclosing the government can make consumers better off.

As we have already pointed, one exception to our results is when, without a minimum standard, the market breaks down and the equilibrium features zero wages with no skilled participation. Because there is always a minimum standard such that an equilibrium exists that features skilled workers, by imposing a standard, the government can create a market that otherwise would not exist. But for this insight to explain why so many states have introduced minimum standards over time, the imposition of the standard should precede the existence of the market. Unfortunately, in most cases, before such standards were introduced, the markets already existed and functioned well.

We chose to focus on the narrow question of whether regulation on disclosure and standards help consumers largely because policy makers use the interests of consumers as justification for the regulation we see. Our paper does not address the wider question of how regulation affects the overall welfare of all participants in the economy. It is possible that regulation on disclosure and standards in all industries is welfare enhancing for the economy as a whole. We leave that unanswered question to future theoretical and empirical research. 


\section{Conclusion}

We have argued in this paper that for professions with a skill in short supply that is in high demand, neither information disclosure nor setting minimum standards improve consumer surplus. Instead they help producers. This raises the question why we observe so much government regulation in practice and whether, in light of our results, the government should adopt a different policy.

It is important to keep in mind that economic models are primarily a means to make a sound argument. This paper should be viewed in this light. It makes the argument that if a government wants to regulate in the interests of consumers, then the implicit assumption made by policy makers, that disclosure and standards help consumers, needs more rigorous justification. What is needed is some economic evidence that the factors we have not modeled in this paper are important enough to overcome the main conclusion of our model, that regulation hurts consumers. In the end, disclosure and standards might indeed be beneficial to consumers, but without supporting evidence, policy makers cannot simply assume that this fact is prima facie true.

To justify regulation, the implication of our model is that policy makers need to make one of the following arguments. They can justify regulating based on Leland (1979), by arguing that the subsequent increase in prices that follows regulation attracts enough new talent to justify the drop in consumer surplus. Not only would this mean better understanding the price-quality elasticity in a sector (that is likely to be quite different in the short term and long term), but also showing that the benefit of the rise in quality outweighs the loss in consumer surplus. Given current government policy and how large this consumer surplus loss can be, we feel that resolving this issue is of first order importance.

A second reason why it might make sense to regulate despite the arguments in this paper is if one believes that consumers do not adequately price the cost of dealing with a charlatan. Here again, evidence should be brought to bear to justify current government policy. As an illustrative example, there has been an increasing number of calls, based the argument that charlatan money managers take advantage of naive investors, for more regulation of the money management industry, with some people suggesting that individuals should be barred from putting their retirement money into active mutual funds. But, in point of fact, there is very little evidence to suggest that mutual fund investors are naive. Quite the contrary, Berk and van Binsbergen (2015) find that investors rationally allocate their capital in this industry. Mutual fund managers that appear to destroy value 
control very little money. Most of the capital in this industry is allocated to high skilled professions. So, in that industry at least, there is little evidence that investor naivete is enough of an issue to warrant regulation.

A third reason why a government might require disclosure is if consumer aversion to the level of uncertainty in producer skill is very large. Because the cost to consumers of being served by a charlatan varies across professions, and as does the ability of consumers to diversify away this risk (by hiring multiple providers), if this was an important driver of government disclosure regulation one would expect to see a relation between the cross sectional variation in these costs and the amount of disclosure. At least, anecdotally, we do not see this variation. For example, government regulators do not appear to have vastly different information disclosure requirements between real estate agents and medical doctors, even though the costs of a mistake are vastly different. Similarly, mutual fund investors can easily diversify away this risk by investing in mutliple funds, but the SEC's disclosure requirements for fund managers are much higher than what is required for medical doctors where such diversification is impossible. ${ }^{8}$

Finally there is the wider question of how regulation affects overall welfare in the economy. Obviously, such a question is very difficult to answer, and we make no claims in this paper to provide insight on it. While consumers make up a large part of economic welfare, we have made no attempt to answer the question of how standards affect the overall supply of skill across sectors in the economy, or how the supply of producers (including charlatans) across sectors affects overall welfare. But with that said, it is premature to simply assume that regulation is welfare enhancing. To justify regulating because it increases overall welfare one would need to provide evidence that other benefits are large enough to outweigh the potentially significant losses to within sector welfare that standards impose.

\footnotetext{
${ }^{8}$ Fund managers are required to reveal their (audited) past perfomance, while doctors are not subject to such a requirement.
} 


\section{References}

Berk, Jonathan B. and Jules H. van Binsbergen, "Measuring skill in the mutual fund industry," Journal of Financial Economics, 2015, 118 (1), 1 - 20.

Egan, Mark, Gregor Matvos, and Amit Seru, "The Market for Financial Adviser Misconduct," Working Paper 22050, National Bureau of Economic Research February 2016.

Hogan, Daniel B, "The effectiveness of licensing: History, evidence, and recommendations.," Law and Human Behavior, 1983, 7 (2-3), 117.

Kleiner, Morris M, "Occupational licensing," The Journal of Economic Perspectives, 2000, 14 (4), 189-202.

- and Alan B Krueger, "Analyzing the extent and influence of occupational licensing on the labor market," Journal of Labor Economics, 2013, 31 (S1), S173-S202.

Kritzer, Herbert M and Neil Vidmar, "When the Lawyer Screws Up: A Portrait of Legal Malpractice Claims and Their Resolution," 2015.

Leland, Hayne E, "Quacks, lemons, and licensing: A theory of minimum quality standards," Journal of Political Economy, 1979, 87 (6), 1328-1346.

Studdert, David M, Marie M Bismark, Michelle M Mello, Harnam Singh, and Matthew J Spittal, "Prevalence and characteristics of physicians prone to malpractice claims," New England Journal of Medicine, 2016, 374 (4), 354-362. 


\section{Appendix}

\section{A Equilibrium}

An equilibrium is a $\{w(\eta), c(\eta), y, \bar{\eta}\}$ with the property that $c(\eta)$ is strictly increasing and thus has a well defined inverse function $\eta(c) \equiv c^{-1}(\cdot)$, such that for every consumer $0 \leq c \leq 1, \eta(c)$ solves the consumer choice problem (3), all skilled agents choose to obtain a signal so that

$$
\int_{\hat{\eta}}^{\infty} w(\eta) f_{s}(\eta) d \eta-A F_{s}(\bar{\eta})>0,
$$

$y>0$ fraction of unskilled agents choose to become charlatans (get a signal) so that

$$
\int_{\bar{\eta}}^{\infty} w(\eta) f_{u}(\eta) d \eta-A F_{u}(\bar{\eta}) \geq 0
$$

and markets clear when agents with signals greater than $\bar{\eta}$ choose to provide the service, that is, for every $\eta$,

$$
\int_{\bar{\eta}}^{\eta} \lambda f_{s}(x)+y f_{u}(x) d x=\int_{\bar{\eta}}^{\eta} d c(x),
$$

and for $\eta<\bar{\eta}, w(\eta)<-A$.

The following proposition derives the conditions under which an equilibrium exists.

Proposition 7 An unique equilibrium exists whenever

$$
\begin{aligned}
& \int_{-\infty}^{\infty} \int_{-\infty}^{\eta} b\left(\lambda F_{s}(v)+(1-\lambda) F_{u}(v)\right)\left(\frac{f_{u}(v) f_{s}^{\prime}(v)-f_{u}^{\prime}(v) f_{s}(v)}{\left(f_{s}(v)+f_{u}(v)\left(\frac{1-\lambda}{\lambda}\right)\right)^{2}}\right) f_{u}(\eta) d v d \eta \\
& \geq A\left(\frac{\lambda}{1-\lambda}\right)
\end{aligned}
$$

with the following properties

$$
\begin{aligned}
w(\eta) & =\int_{\bar{\eta}}^{\eta} b(c(v)) \pi^{\prime}(v) d v-A \\
c(\eta) & =1-\lambda\left(1-F_{s}(\eta)\right)-y\left(1-F_{u}(\eta)\right)
\end{aligned}
$$

and $y=1$ when

$$
\int_{\bar{\eta}}^{\infty} \int_{\bar{\eta}}^{\eta} b(c(v)) \pi^{\prime}(v) f_{u}(\eta) d v d \eta \geq A
$$


and $\bar{\eta}$ solves

$$
\lambda F_{s}(\bar{\eta})+F_{u}(\bar{\eta})=\lambda
$$

otherwise

$$
y=\frac{1-\lambda\left(1-F_{s}(\bar{\eta})\right)}{1-F_{u}(\bar{\eta})}
$$

and $\bar{\eta}$ solves

$$
\int_{\bar{\eta}}^{\infty} \int_{\bar{\eta}}^{\eta} b(c(v)) \pi^{\prime}(v) f_{u}(\eta) d v d \eta=A .
$$

Proof. We begin by first solving the consumer choice problem. Consumer $c$ picks her provider based on the provider's signal, $\eta$, by maximizing the benefit of consuming, that is,

$$
\max _{\eta}(b(c) \pi(\eta)-w(\eta)) .
$$

The solution, $\eta^{*}$ to this problem, assuming it exists, solves

$$
w^{\prime}\left(\eta^{*}\right)=b(c) \pi^{\prime}\left(\eta^{*}\right) .
$$

Define the function $\eta(c):[0,1] \rightarrow(-\infty, \infty)$ as the solution to this equation for each consumer $c$. As we show shortly, $\eta(c)$ is strictly increasing, so we can define the inverse function, $c(\cdot)=\eta^{-1}(\cdot)$, that is, for each signal realization the consumer who chooses to consume at that realization. Using this inverse function, (20) can be written as

$$
w^{\prime}(\eta)=b(c(\eta)) \pi^{\prime}(\eta)
$$

Integrating (21) gives

$$
w(\eta)=\int_{-\infty}^{\eta} b(c(v)) \pi^{\prime}(v) d v+C
$$

where $C$ is the constant of integration which is determined to ensure that markets clear. Let $\bar{\eta}$ be the critical signal such that, if a producer will only choose to provide the service if he gets a signal greater than $\bar{\eta}$ and consumer only choose to consume the skill if the signal exceeds $\bar{\eta}$. This condition implies that a producer with signal $\bar{\eta}$ must be indifferent between supplying the skill or working for the reservation wage:

$$
w(\bar{\eta})=\int_{-\infty}^{\bar{\eta}} b(c(v)) \pi^{\prime}(v) d v+C=-A
$$

so

$$
C=-\int_{-\infty}^{\bar{\eta}} b(c(v)) \pi^{\prime}(v) d u-A
$$


So substituting (23) into (22) gives an explicit expression for the wage:

$$
\begin{aligned}
w(\eta) & =\int_{-\infty}^{\eta} b(c(v)) \pi^{\prime}(v) d v-\int_{-\infty}^{\bar{\eta}} b(c(v)) \pi^{\prime}(v) d u-A \\
& =\int_{\bar{\eta}}^{\eta} b(c(v)) \pi^{\prime}(v) d u-A
\end{aligned}
$$

For markets to clear, for every $\eta$ the supply of people providing the skill must equal the demand:

$$
\int_{\bar{\eta}}^{\eta} \lambda f_{s}(x)+y f_{u}(x) d x=\int_{\bar{\eta}}^{\eta} d c(x) .
$$

where $y$ is the fraction of unskilled agents that choose to become charlatans (i.e., choose to enter the market as a producer and get a signal). Taking derivatives in (25) gives:

$$
c^{\prime}(\eta)=\lambda f_{s}(\eta)+y f_{u}(\eta)
$$

Integrating provides the function

$$
c(\eta)=\lambda F_{s}(\eta)+y F_{u}(\eta)-\lambda+1-y
$$

where the final term, the constant of integration, is determined by the boundary condition that the consumer with the largest $b$ chooses to consume the largest signal, $c(\infty)=1$. The function $c(\eta)$ is strictly increasing because $F_{s}(\eta)$ and $F_{u}(\eta)$ are strictly increasing. Notice that $w(\bar{\eta})=-A \leq 0$, implying $b(c) \pi(\bar{\eta})-w(\bar{\eta}) \geq 0$ for every $c$, so every (unskilled) agent will choose to consume implying, $c(\bar{\eta})=0$. Substituting this condition into (26) gives:

$$
\lambda F_{s}(\bar{\eta})+y F_{u}(\bar{\eta})=\lambda-1+y .
$$

When $y=1$ we have (17). Otherwise,

$$
y=\frac{1-\lambda\left(1-F_{s}(\bar{\eta})\right)}{1-F_{u}(\bar{\eta})}
$$

Note that (28) implies that $y \geq 1-\lambda$.

Next we need to prove existence and uniqueness of the solution to the consumer choice problem. Our first order of business is to show that consumers second order condition is satisfied, that is, that

$$
w^{\prime \prime}(\eta)-b(s) \pi^{\prime \prime}(\eta)>0
$$


From (21)

$$
w^{\prime \prime}(\eta)=b^{\prime}(c(\eta)) \pi^{\prime}(\eta) c^{\prime}(\eta)+b(c(\eta)) \pi^{\prime \prime}(\eta)
$$

so

$$
w^{\prime \prime}(\eta)-b(s) \pi^{\prime \prime}(\eta)=b^{\prime}(c(\eta)) \pi^{\prime}(\eta) c^{\prime}(\eta)+b(c(\eta)) \pi^{\prime \prime}(\eta)-b(s) \pi^{\prime \prime}(\eta) .
$$

Evaluating this expression at the optimal, $\eta(s)$ gives

$$
w^{\prime \prime}(\eta(s))-b(s) \pi^{\prime \prime}(\eta(s))=b^{\prime}(s) \pi^{\prime}(\eta(s)) c^{\prime}(\eta(s))>0
$$

because all three functions are strictly increasing and so have strictly positive derivatives, so the condition is always satisfied at $\eta(s)$. In fact, the solution is unique, that is, $\eta(s)$ is also a global maximum. To see why note that the slope of $b(s) \pi(\eta)-w(\eta)$ is given by

$$
b(s) \pi^{\prime}(\eta)-w^{\prime}(\eta)=\left(b(s)-b(c(\eta)) \pi^{\prime}(\eta)\right.
$$

so the sign is the sign of the first term. Because both $b(s)$ and $c(\eta)$ are increasing functions, the slope is negative for all $\eta>\eta(s)$ and positive for all $\eta<\eta(s)$, which implies that $\eta^{*}$ is the global maximum and so is the unique solution.

Finally we need to show that all skilled agents and $y$ fraction of unskilled agents will choose to produce. The expected benefit for an unskilled agent to produce is

$$
\begin{aligned}
\int_{\bar{\eta}}^{\infty} w(\eta) f_{u}(\eta) d \eta-A F_{u}(\bar{\eta}) & =\int_{\bar{\eta}}^{\infty} \int_{\bar{\eta}}^{\eta} b(c(v)) \pi^{\prime}(v) f_{u}(\eta) d v d \eta-A\left(1-F_{u}(\bar{\eta})\right)-A F_{u}(\bar{\eta}) \\
& =\int_{\bar{\eta}}^{\infty} \int_{\bar{\eta}}^{\eta} b(c(v)) \pi^{\prime}(v) f_{u}(\eta) d v d \eta-A
\end{aligned}
$$

There are two cases we need to consider, the case when the equilibrium has $y=1$ and when $y<1$. Let $\bar{\eta}^{*}$ solve (27) with $y=1$. Note that a unique solution exists because the left hand side of (27) is continuous, strictly increasing, and is strictly larger than $\lambda$ when $\bar{\eta}=\infty$ and less than $\lambda$ when $\bar{\eta}=-\infty$. If (29) is positive at $\bar{\eta}^{*}$, then all unskilled agents will choose to produce and $\bar{\eta}=\bar{\eta}^{*}$. If (29) is not positive with $y=1$ and $\bar{\eta}=\bar{\eta}^{*}$, then an equilibrium, if it exists, features $y<1$. Let $\bar{\eta}=-\infty$ so that, from (28),

$$
y=1-\lambda .
$$


Using this expression for $y$ and substituting $\bar{\eta}=-\infty$ into (29) gives

$$
\left(\frac{1-\lambda}{\lambda}\right) \int_{-\infty}^{\infty} \int_{-\infty}^{\eta} b\left(\lambda F_{s}(v)+(1-\lambda) F_{u}(v)\right)\left(\frac{f_{u}(v) f_{s}^{\prime}(v)-f_{u}^{\prime}(v) f_{s}(v)}{\left(f_{s}(v)+f_{u}(v)\left(\frac{1-\lambda}{\lambda}\right)\right)^{2}}\right) f_{u}(\eta) d v d \eta-A \geq 0
$$

where the inequality follows from (16). Because (29) is a continuous function and is smaller than or equal to zero when $\bar{\eta}=\bar{\eta}^{*}$ and equal to or greater than zero when $\bar{\eta}=-\infty$, there must exist a $-\infty \leq \bar{\eta}<\bar{\eta}^{*}$ such that (29) equals zero (i.e., (19) is satisfied), implying that an equilibrium exists with $y<1$. We have shown that if (16) holds, a $\bar{\eta}$ always exists such that

$$
\int_{\bar{\eta}}^{\infty} w(\eta) f_{u}(\eta) d \eta-A F_{u}(\bar{\eta}) \geq 0
$$

implying that a fraction $y$ of unskilled agents will choose to produce. Because $F_{s}$ stochastically dominates $F_{u}$, at this $\bar{\eta}$,

$$
\int_{\bar{\eta}}^{\infty} \int_{\bar{\eta}}^{\eta} b(c(v)) \pi^{\prime}(v) f_{s}(\eta) d v d \eta>\int_{\bar{\eta}}^{\infty} \int_{\bar{\eta}}^{\eta} b(c(v)) \pi^{\prime}(v) f_{u}(\eta) d v d \eta
$$

implying that all skilled agents will also produce.

\section{B Equilibrium with a Minimum Standard}

Let $\Delta$ solve

$$
\lambda F_{s}(\Delta)+F_{u}(\Delta)=\lambda
$$

An equilibrium in an economy with a minimum standard $\hat{\eta} \geq \Delta$ is a $\{w(\eta), c(\eta), y\}$ with the property that $c(\eta)$ is strictly increasing and thus has a well defined inverse function $\eta(c) \equiv c^{-1}(\cdot)$, such that for every consumer $0 \leq c \leq 1, \eta(c)$ solves the consumer choice problem (3), all skilled agents choose to obtain a signal so that

$$
\int_{\hat{\eta}}^{\infty} w(\eta) f_{s}(\eta) d \eta-A F_{s}(\bar{\eta})>0
$$

either $y>0$ fraction of unskilled agents choose to become charlatans (get a signal) so that

$$
\int_{\hat{\eta}}^{\infty} w(\eta) f_{u}(\eta) d \eta-A F_{u}(\bar{\eta}) \geq 0
$$


or no unskilled agent chooses to become a charlatan $(y=0)$ so that

$$
\int_{\hat{\eta}}^{\infty} w(\eta) f_{u}(\eta) d \eta-A F_{u}(\bar{\eta})<0
$$

and markets clear with agents with signals greater than $\hat{\eta}$ always choosing to provide the service, that is, for every $\eta$,

$$
\int_{\hat{\eta}}^{\eta} \lambda f_{s}(x)+y(1-\lambda) f_{u}(x) d x=(1-\lambda) \int_{\hat{\eta}}^{\eta} d c(x) .
$$

The following proposition shows that when a minimum standard is introduced such that $\hat{\eta} \geq \Delta$, an equilibrium always exists. The equilibrium is characterized by three regions corresponding to the fraction of unskilled agents who choose to become charlatans.

Proposition 8 When a minimum standard $\hat{\eta} \geq \Delta$ is imposed such that only producers who get a signal $\eta \geq \hat{\eta}$ are allowed to produce, an equilibrium always exists with the following properties:

$$
\begin{aligned}
c(\eta)= & 1-\lambda\left(1-F_{s}(\eta)\right)-y\left(1-F_{u}(\eta)\right) \\
w(\eta)= & \int_{\hat{\eta}}^{\eta} b(c(v)) \pi^{\prime}(v) d v+b(c(\hat{\eta})) \pi(\hat{\eta}) \\
= & \left(\frac{y}{\lambda}\right) \int_{\hat{\eta}}^{\eta} b(c(v))\left(\frac{f_{u}(v) f_{s}^{\prime}(v)-f_{u}^{\prime}(v) f_{s}(v)}{\left(f_{s}(v)+f_{u}(v)\left(\frac{y}{\lambda}\right)\right)^{2}}\right) d u \\
& +b(c(\hat{\eta}))\left(\frac{f_{s}(\hat{\eta})}{f_{s}(\hat{\eta})+f_{u}(\hat{\eta})\left(\frac{y}{\lambda}\right)}\right) .
\end{aligned}
$$

and

$$
y=\left\{\begin{array}{lrr}
1 & \text { if } \quad \int_{\hat{\eta}}^{\infty} \int_{\hat{\eta}}^{\eta} b\left(\lambda F_{s}(v)+F_{u}(v)-\lambda\right) \pi^{\prime}(v) f_{u}(\eta) d v d \eta+ \\
& b\left(\lambda F_{s}(v)+F_{u}(v)-\lambda\right) \pi(\hat{\eta})\left(1-F_{u}(\hat{\eta})\right)-A F_{u}(\hat{\eta})>0 \\
0 & \text { if } \quad b\left(1-\lambda\left(1-F_{s}(\hat{\eta})\right)\right)\left(1-F_{u}(\hat{\eta})\right)-A F_{u}(\hat{\eta})<0 \\
y(\hat{\eta}) \quad & \text { o.w. }
\end{array}\right.
$$


where $y(\hat{\eta})$ solves the following equation

$$
\begin{aligned}
& \int_{\hat{\eta}}^{\infty} \int_{\hat{\eta}}^{\eta} b\left(1-\lambda\left(1-F_{s}(v)\right)-y(\hat{\eta})\left(1-F_{u}(v)\right)\right)\left(\left(\frac{y(\hat{\eta})}{\lambda}\right) \frac{f_{u}(v) f_{s}^{\prime}(v)-f_{u}^{\prime}(v) f_{s}(v)}{\left(f_{s}(v)+f_{u}(v)\left(\frac{y(\hat{\eta})}{\lambda}\right)\right)^{2}}\right) f_{u}(\eta) d v d \eta \\
& \quad=A F_{u}(\hat{\eta})-b\left(1-\lambda\left(1-F_{s}(\hat{\eta})\right)-y(\hat{\eta})\left(1-F_{u}(\hat{\eta})\right)\right)\left(\frac{f_{s}(\hat{\eta})}{f_{s}(\hat{\eta})+f_{u}(\hat{\eta})\left(\frac{y(\hat{\eta})}{\lambda}\right)}\right)\left(1-F_{u}(\hat{\eta})\right)
\end{aligned}
$$

Proof. We begin by deriving $c(\eta)$ from the market clearing condition. Using the same logic as in the proof of Proposition 7, we get

$$
c(\eta)=\lambda F_{s}(\eta)+y F_{u}(\eta)-\lambda+1-y
$$

Note that $0 \leq c(\eta) \leq 1$ so long as $\eta \geq \Delta$. Next we derive wages. When $y>0$ the wage function is also derived using the same logic as in the proof of Proposition 7 providing

$$
w(\eta)=\int_{-\infty}^{\eta} b(c(v)) \pi^{\prime}(v) d v+C .
$$

The constant $C$ is now determined by the condition that $c(\hat{\eta})$ must be indifferent between consuming or not consuming (so that all consumers $c<c(\hat{\eta})$ choose not to consume):

$$
b(c(\hat{\eta})) \pi(\hat{\eta})-w(\hat{\eta})=0 .
$$

Substituting for wages using (35) and rearranging terms gives,

$$
C=b(c(\hat{\eta})) \pi(\hat{\eta})-\int_{-\infty}^{\hat{\eta}} b(c(v)) \pi^{\prime}(v) d v
$$

Substituting this into (35) gives the wage function

$$
w(\eta)=\int_{\hat{\eta}}^{\eta} b(c(v)) \pi^{\prime}(v) d u+b(c(\hat{\eta})) \pi(\hat{\eta})
$$

An unskilled worker will choose to produce (become a charlatan) if the expected wage is greater then zero:

$$
-A F_{u}(\hat{\eta})+\int_{\hat{\eta}}^{\infty} w(\eta) f_{u}(\eta) d \eta \geq 0
$$


If this condition holds strictly, $y=1$. Substituting (36) into (37) provides the condition in (32) for $y=1$ in the statement of the proposition. So we have derived the first condition in $(32)$.

Next consider the case when $y=0$. In this case there are no charlatans so the signal becomes relevant only in that it must exceed $\hat{\eta}$. All signals that exceed $\hat{\eta}$ are uninformative because all workers are skilled and therefore earn the same wage. The wage is determined by the condition that $c(\hat{\eta})$ is indifferent between consuming or not:

$$
w(\eta)=b(c(\hat{\eta}))
$$

which is what $(31)$ reduces to when $y=0$. In this case unskilled workers prefer not to produce, so

$$
-A F_{u}(\hat{\eta})+\int_{\hat{\eta}}^{\infty} w(\eta) f_{u}(\eta) d \eta<0
$$

Substituting (38) into (39) provides the condition in (32) for $y=0$ in the statement of the proposition. So we have derived the second condition in (32).

Finally, $0<y<1$ can only occur when

$$
-A F_{u}(\hat{\eta})+\int_{\hat{\eta}}^{\infty} w(\eta) f_{u}(\eta) d \eta=0
$$

Substituting (36) into this expression gives

$$
-A F_{u}(\hat{\eta})+\int_{\hat{\eta}}^{\infty} \int_{\hat{\eta}}^{\eta} b(c(v)) \pi^{\prime}(v) f_{u}(\eta) d v d \eta+b(c(\hat{\eta})) \pi(\hat{\eta})\left(1-F_{u}(\hat{\eta})\right)=0
$$

which is (33).

To establish existence we need to show that if the first two conditions in (32) are not satisfied, (33) will always have a solution. Notice that if the condition in (32) for $y=1$ fails the left hand side of $(33)$ with $y(\hat{\eta})=1$ is less than or equal to zero. Similarly, if the condition in $(32)$ for $y=0$ fails the left hand side of $(33)$ with $y(\hat{\eta})=0$ is greater than or equal to zero. Since the left hand side of (33) is a continuous function, by the intermediate value theorem, there must be a value of $y(\hat{\eta})$ that sets the left hand side equal to zero.

Proposition 9 In an economy in which a minimum standard $\hat{\eta}<\Delta$ is imposed such that 
only producers who get a signal $\eta \geq \hat{\eta}$ are allowed to produce, if

$$
\int_{\hat{\eta}}^{\infty} \int_{\hat{\eta}}^{\eta} b(c(v)) \pi^{\prime}(v) f_{u}(\eta) d v d \eta \leq A \leq\left(\frac{1}{F_{u}(\hat{\eta})}\right) \int_{\hat{\eta}}^{\infty} \int_{\hat{\eta}}^{\eta} b(c(v)) \pi^{\prime}(v) f_{u}(\eta) d v d \eta
$$

where

$$
c(\eta)=1-\lambda\left(1-F_{s}(\eta)\right)-y\left(1-F_{u}(\eta)\right)
$$

and

$$
y=\frac{1-\lambda\left(1-F_{s}(\hat{\eta})\right)}{1-F_{u}(\hat{\eta})}
$$

then an equilibrium exists with

$$
w(\eta)=\int_{\hat{\eta}}^{\eta} b(c(v)) \pi^{\prime}(v) d u-\left(\frac{1}{1-F_{u}(\hat{\eta})}\right) \int_{\hat{\eta}}^{\infty} \int_{\hat{\eta}}^{x} b(c(v)) \pi^{\prime}(v) d v f_{u}(x) d x+A\left(\frac{F_{u}(\hat{\eta})}{1-F_{u}(\hat{\eta})}\right) .
$$

Proof. We begin by deriving $c(\eta)$ from market clearing condition. Using the same logic as in the proof of Proposition 7, we get

$$
c(\eta)=1-\lambda\left(1-F_{s}(\eta)\right)-y\left(1-F_{u}(\eta)\right)
$$

Notice that $\hat{\eta}<\Delta$ implies that if $y=1$ then supply would exceed demand. That implies that $y<1$. At the equilibrium $y, c(\hat{\eta})=0$ because $\hat{\eta}>\bar{\eta}$. Using this condition to solve (44) for $y$ gives:

$$
y=\frac{1-\lambda\left(1-F_{s}(\hat{\eta})\right)}{1-F_{u}(\hat{\eta})}<1
$$

Using the same logic as in the proof of Proposition 7, the wage function is

$$
w(\eta)=\int_{\hat{\eta}}^{\eta} b(c(v)) \pi^{\prime}(v) d v+C .
$$

Because $c(\hat{\eta})=0$, all consumers must either be indifferent or strictly prefer to consume, so wages are not determined by the consumer's indifference condition. Instead, the constant $C$ is now determined by the condition that because $y<1$, unskilled producers must be indifferent about deciding whether to produce:

$$
-A F_{u}(\hat{\eta})+\int_{\hat{\eta}}^{\infty} w(\eta) f_{u}(\eta) d \eta=0 .
$$


Substituting for wages using (46) and rearranging terms gives,

$$
\begin{aligned}
& -A F_{u}(\hat{\eta})+\int_{\hat{\eta}}^{\infty} \int_{\hat{\eta}}^{\eta} b(c(v)) \pi^{\prime}(v) d v f_{u}(\eta) d \eta+C\left(1-F_{u}(\hat{\eta})\right)=0 \\
& C=A\left(\frac{F_{u}(\hat{\eta})}{1-F_{u}(\hat{\eta})}\right)-\left(\frac{1}{1-F_{u}(\hat{\eta})}\right) \int_{\hat{\eta}}^{\infty} \int_{\hat{\eta}}^{\eta} b(c(v)) \pi^{\prime}(v) d v f_{u}(\eta) d \eta .
\end{aligned}
$$

Substituting this into (46) gives the wage function

$$
w(\eta)=\int_{\hat{\eta}}^{\eta} b(c(v)) \pi^{\prime}(v) d u-\left(\frac{1}{1-F_{u}(\hat{\eta})}\right) \int_{\hat{\eta}}^{\infty} \int_{\hat{\eta}}^{x} b(c(v)) \pi^{\prime}(v) d v f_{u}(x) d x+A\left(\frac{F_{u}(\hat{\eta})}{1-F_{u}(\hat{\eta})}\right) .
$$

Now

$$
w(\hat{\eta})=A\left(\frac{F_{u}(\hat{\eta})}{1-F_{u}(\hat{\eta})}\right)-\left(\frac{1}{1-F_{u}(\hat{\eta})}\right) \int_{\hat{\eta}}^{\infty} \int_{\hat{\eta}}^{x} b(c(v)) \pi^{\prime}(v) d v f_{u}(x) d x \leq 0
$$

by (42). This implies that $b(c) \pi(\hat{\eta})-w(\hat{\eta}) \geq 0$ for every $c$, verifying that $c(\hat{\eta})=0$. Finally, producers must be willing to produce. Using (42)

$$
\begin{aligned}
w(\hat{\eta}) & =A\left(\frac{F_{u}(\hat{\eta})}{1-F_{u}(\hat{\eta})}\right)-\left(\frac{1}{1-F_{u}(\hat{\eta})}\right) \int_{\hat{\eta}}^{\infty} \int_{\hat{\eta}}^{x} b(c(v)) \pi^{\prime}(v) d v f_{u}(x) d x \\
& \geq A\left(\frac{F_{u}(\hat{\eta})}{1-F_{u}(\hat{\eta})}\right)-\left(\frac{1}{1-F_{u}(\hat{\eta})}\right) A \\
& =-A
\end{aligned}
$$

so all producers are better off producing rather than earning the reservation wage. 


\section{Proof of Proposition 1}

Consumer surplus in the equilibrium without a standard is

$$
\begin{aligned}
\int_{\bar{\eta}}^{\infty} & b(c(\eta)) \pi(\eta)-b(c(\eta)) \pi(\eta)+\lambda \int_{\bar{\eta}}^{\eta} b^{\prime}(c(v)) f_{s}(v) d v+A d c(\eta) \\
= & \lambda \int_{\bar{\eta}}^{\infty} \int_{\bar{\eta}}^{\eta} b^{\prime}(c(v)) f_{s}(v) d v d c(\eta)+A \\
= & \lambda \int_{\bar{\eta}}^{\infty} \int_{\bar{\eta}}^{\eta} b^{\prime}(c(v)) f_{s}(v) d v\left(\lambda f_{s}(\eta)+y f_{u}(\eta)\right) d \eta+A \\
= & \lambda \int_{\bar{\eta}}^{\infty} \int_{(\bar{\eta}-1) / \sigma}^{(\eta-1) / \sigma} b^{\prime}(c(u \sigma+1)) n(u) d u\left(\lambda f_{s}(\eta)+y f_{u}(\eta)\right) d \eta+A \\
= & \lambda \int_{\bar{\eta} / \sigma}^{\infty} \int_{(\bar{\eta}-1) / \sigma}^{v-1 / \sigma} b^{\prime}(c(u \sigma+1)) n(u) d u(\lambda n(v-1 / \sigma)+y n(v)) d v+A
\end{aligned}
$$

where $n(x)$ is a standard normal density function. Define $N(x)$ to be the standard normal distribution function. We first consider the case where $y=1$ so $y$ does not depend on $\sigma$. Taking the derivative of (48) w.r.t. $\sigma$ gives

$$
\begin{aligned}
\frac{d}{d \sigma}\left(\lambda \int_{\bar{\eta} / \sigma}^{\infty} \int_{(\bar{\eta}-1) / \sigma}^{v-1 / \sigma} b^{\prime}(c(u \sigma+1)) n(u) d u(\lambda n(v-1 / \sigma)+y n(v)) d v+A\right) \\
=\quad \lambda \int_{\bar{\eta} / \sigma}^{\infty} \frac{d}{d \sigma} \int_{(\bar{\eta}-1) / \sigma}^{v-1 / \sigma} b^{\prime}(c(u \sigma+1)) n(u) d u(\lambda n(v-1 / \sigma)+y n(v)) d v \\
=\frac{\lambda}{\sigma^{2}} \int_{\bar{\eta} / \sigma}^{\infty} b^{\prime}(c(v \sigma)) n(v-1 / \sigma)(\lambda n(v-1 / \sigma)+y n(v)) d v \\
\quad-\frac{\lambda}{\sigma} b^{\prime}(c(\bar{\eta})) n\left(\frac{\bar{\eta}-1}{\sigma}\right)\left(\frac{\partial \bar{\eta}}{\partial \sigma}-\frac{\bar{\eta}-1}{\sigma}\right) \\
+\lambda \int_{\bar{\eta} / \sigma}^{\infty} \int_{(\bar{\eta}-1) / \sigma}^{v-1 / \sigma} b^{\prime \prime}(c(u \sigma+1)) \frac{d c(u \sigma+1)}{d \sigma} n(u) d u(\lambda n(v-1 / \sigma)+y n(v)) d v \\
\quad-\frac{\lambda^{2}}{\sigma^{2}} \int_{\bar{\eta} / \sigma}^{\infty} \int_{(\bar{\eta}-1) / \sigma}^{v-1 / \sigma} b^{\prime}(c(u \sigma+1)) n(u) d u(v-1 / \sigma) n(v-1 / \sigma) d v \\
=\frac{\lambda y}{\sigma^{2}} \int_{\bar{\eta} / \sigma}^{\infty} b^{\prime}(c(v \sigma)) n(v-1 / \sigma) n(v) d v \\
\quad-\frac{\lambda}{\sigma} b^{\prime}(c(\bar{\eta})) n\left(\frac{\bar{\eta}-1}{\sigma}\right)\left(\frac{\partial \bar{\eta}}{\partial \sigma}-\frac{\bar{\eta}-1}{\sigma}\right) \\
\quad-\frac{\lambda y^{2}}{\sigma^{2}} \int_{\bar{\eta} / \sigma}^{\infty} \int_{(\bar{\eta}-1) / \sigma}^{v-1 / \sigma} b^{\prime \prime}(c(u \sigma+1)) n(u+1 / \sigma) n(u) d u n(v) d v+o\left(\lambda^{2}\right)
\end{aligned}
$$


because from (26) we have

$c(u \sigma+1)=1-\lambda\left(1-F_{s}(u \sigma+1)\right)-y\left(1-F_{u}(u \sigma+1)\right)=1-\lambda(1-N(u))-y(1-N(u+1 / \sigma))$

SO

$$
\frac{d}{d \sigma} c(u \sigma+1)=-\frac{y}{\sigma^{2}} n(u+1 / \sigma) .
$$

Now because $c(\bar{\eta})=0$ we have

$$
1-\lambda\left(1-F_{s}(\bar{\eta})\right)-y\left(1-F_{u}(\bar{\eta})\right)=0
$$

Taking derivatives gives

$$
\begin{gathered}
\lambda \frac{d}{d \sigma} F_{s}(\bar{\eta})+y \frac{d}{d \sigma} F_{u}(\bar{\eta})=0 \\
\lambda \frac{\partial}{\partial \sigma} F_{s}(\bar{\eta})+y \frac{\partial}{\partial \sigma} F_{u}(\bar{\eta})+\lambda f_{s}(\bar{\eta}) \frac{\partial \bar{\eta}}{\partial \sigma}+y f_{u}(\bar{\eta}) \frac{\partial \bar{\eta}}{\partial \sigma}=0 \\
\frac{\partial \bar{\eta}}{\partial \sigma}=-\frac{\lambda \frac{\partial}{\partial \sigma} F_{s}(\bar{\eta})+y \frac{\partial}{\partial \sigma} F_{u}(\bar{\eta})}{\lambda f_{s}(\bar{\eta})+y f_{u}(\bar{\eta})}<0
\end{gathered}
$$

Under the normal assumption, (52) becomes

$$
\frac{\partial \bar{\eta}}{\partial \sigma}=-\frac{\lambda \frac{\partial}{\partial \sigma} F_{s}(\bar{\eta})+y \frac{\partial}{\partial \sigma} F_{u}(\bar{\eta})}{\lambda f_{s}(\bar{\eta})+y f_{u}(\bar{\eta})}=\frac{\bar{\eta}-\pi(\bar{\eta})}{\sigma}
$$


Substituting this expression into (49) and using the fact that $b^{\prime \prime}(\cdot)<0$ gives,

$$
\begin{aligned}
\frac{d}{d \sigma}\left(\lambda \int_{\bar{\eta} / \sigma}^{\infty} \int_{(\bar{\eta}-1) / \sigma}^{v-1 / \sigma} b^{\prime}(c(u \sigma+1)) n(u) d u(\lambda n(v-1 / \sigma)+y n(v)) d v+A\right) \\
>\quad \frac{\lambda y}{\sigma^{2}} \int_{\bar{\eta} / \sigma}^{\infty} b^{\prime}(c(v \sigma)) n(v-1 / \sigma) n(v) d v \\
\quad-\frac{\lambda}{\sigma} b^{\prime}(c(\bar{\eta})) n\left(\frac{\bar{\eta}-1}{\sigma}\right)\left(\frac{\partial \bar{\eta}}{\partial \sigma}-\frac{\bar{\eta}-1}{\sigma}\right)+o\left(\lambda^{2}\right) \\
=\frac{\lambda y}{\sigma^{2}} \int_{\bar{\eta} / \sigma}^{\infty} b^{\prime}(c(v \sigma)) n(v-1 / \sigma) n(v) d v \\
\quad-\frac{\lambda}{\sigma} b^{\prime}(c(\bar{\eta})) n\left(\frac{\bar{\eta}-1}{\sigma}\right)\left(\frac{1-\pi(\bar{\eta})}{\sigma}\right)+o\left(\lambda^{2}\right) \\
>\quad \frac{\lambda y}{\sigma^{2}} \int_{\bar{\eta} / \sigma}^{\infty} b^{\prime}(c(v \sigma)) n(v-1 / \sigma) n(v) d v \\
\quad-\frac{\lambda}{\sigma} b^{\prime}(c(\bar{\eta})) n\left(\frac{\bar{\eta}-1}{\sigma}\right)\left(\frac{1}{\sigma}\right)+o\left(\lambda^{2}\right) \\
>\quad \frac{\lambda y}{\sigma^{2}} \int_{\bar{\eta} / \sigma}^{\infty} b^{\prime}(c(v \sigma)) n(v-1 / \sigma) n(v) d v-\frac{\lambda}{\sigma} b^{\prime}(c(\bar{\eta})) n\left(\frac{\bar{\eta}-1}{\sigma}\right)\left(\frac{1}{\sigma}\right)+o\left(\lambda^{2}\right) .
\end{aligned}
$$

Note, from Proposition 7 that $y \geq 1-\lambda$. Substituting this, and integrating the first term in (53) gives

$$
\begin{aligned}
& \frac{\lambda y}{\sigma^{2}} \int_{\bar{\eta} / \sigma}^{\infty} b^{\prime}(c(v \sigma)) n(v-1 / \sigma) n(v) d v-\frac{\lambda}{\sigma^{2}} b^{\prime}(c(\bar{\eta})) n\left(\frac{\bar{\eta}-1}{\sigma}\right)+o\left(\lambda^{2}\right) \\
& \quad \geq \frac{\lambda}{\sigma^{2}} \frac{e^{-\frac{1}{2 \sigma^{2}}}}{\sqrt{2 \pi}} \int_{\bar{\eta} / \sigma}^{\infty} b^{\prime}(c(v \sigma)) n\left(\sqrt{2}\left(v-\frac{1}{2 \sigma}\right)\right) d v-\frac{\lambda}{\sigma^{2}} b^{\prime}(c(\bar{\eta})) n\left(\frac{\bar{\eta}-1}{\sigma}\right)+o\left(\lambda^{2}\right) \\
& >\frac{\lambda}{\sigma^{2}} \frac{e^{-\frac{1}{2 \sigma^{2}}}}{\sqrt{2 \pi}} b^{\prime}(1)\left(1-N\left(\frac{2 \bar{\eta}-1}{\sqrt{2} \sigma}\right)\right)-\frac{\lambda}{\sigma^{2}} b^{\prime}(c(\bar{\eta})) n\left(\frac{\bar{\eta}-1}{\sigma}\right)+o\left(\lambda^{2}\right) \\
& =\frac{\lambda}{\sigma^{2}}\left(\frac{e^{-\frac{1}{2 \sigma^{2}}}}{\sqrt{2 \pi}} b^{\prime}(1)\left(1-N\left(\frac{2 \bar{\eta}-1}{\sqrt{2} \sigma}\right)\right)-b^{\prime}(0) n\left(\frac{\bar{\eta}-1}{\sigma}\right)\right)+o\left(\lambda^{2}\right)
\end{aligned}
$$


For small enough $\lambda$, this expression is positive so long as

$$
\begin{aligned}
& \frac{e^{-\frac{1}{2 \sigma^{2}}}}{\sqrt{2 \pi}}\left(\frac{b^{\prime}(1)}{b^{\prime}(0)}\right)\left(1-N\left(\frac{2 \bar{\eta}-1}{\sqrt{2} \sigma}\right)\right)>\frac{e^{-\frac{1}{2}\left(\frac{\bar{\eta}-1}{\sigma}\right)^{2}}}{\sqrt{2 \pi}} \\
&-\frac{1}{2 \sigma^{2}}+\log \left(\left(\frac{b^{\prime}(1)}{b^{\prime}(0)}\right)\left(1-N\left(\frac{2 \bar{\eta}-1}{\sqrt{2} \sigma}\right)\right)\right)>-\frac{1}{2}\left(\frac{\bar{\eta}-1}{\sigma}\right)^{2} \\
& 1-2 \sigma^{2} \log \left(\left(\frac{b^{\prime}(1)}{b^{\prime}(0)}\right)\left(1-N\left(\frac{2 \bar{\eta}-1}{\sqrt{2} \sigma}\right)\right)\right)<(\bar{\eta}-1)^{2} \\
& \sqrt{1-2 \sigma^{2} \log \left(\left(\frac{b^{\prime}(1)}{b^{\prime}(0)}\right)\left(1-N\left(\frac{2 \bar{\eta}-1}{\sqrt{2} \sigma}\right)\right)\right)}<\bar{\eta}-1 \text { or } \\
& \sqrt{1-2 \sigma^{2} \log \left(\left(\frac{b^{\prime}(1)}{b^{\prime}(0)}\right)\left(1-N\left(\frac{2 \bar{\eta}-1}{\sqrt{2} \sigma}\right)\right)\right)}<-\bar{\eta}+1
\end{aligned}
$$

Using the second inequality gives

$$
\begin{aligned}
\bar{\eta} & <1-\sqrt{1+2 \sigma^{2} \log \left(\frac{b^{\prime}(0)}{b^{\prime}(1)\left(1-N\left(\frac{2 \bar{\eta}-1}{\sqrt{2} \sigma}\right)\right)}\right)} \\
& <1-\sqrt{1+2 \sigma^{2} \log \left(\frac{b^{\prime}(0)}{b^{\prime}(1)}\right)}
\end{aligned}
$$

From (51) we have

$$
\lambda=\frac{1-y\left(1-N\left(\frac{\bar{\eta}}{\sigma}\right)\right)}{1-N\left(\frac{\bar{\eta}-1}{\sigma}\right)} \geq \frac{N\left(\frac{\bar{\eta}}{\sigma}\right)}{1-N\left(\frac{\bar{\eta}-1}{\sigma}\right)}
$$

which implies that there exists a $\lambda^{*}$ such that for all $\lambda<\lambda^{*},(55)$ is satisfied. That implies that there exists a $\hat{\lambda} \leq \lambda^{*}$ such that for all $\lambda<\hat{\lambda}$ where an equilibrium exists,

$$
\frac{d}{d \sigma}\left(\lambda \int_{\bar{\eta} / \sigma}^{\infty} \int_{(\bar{\eta}-1) / \sigma}^{v-1 / \sigma} b^{\prime}(c(u \sigma+1)) n(u) d u(\lambda n(v-1 / \sigma)+y n(v)) d v+A\right)>0 .
$$

The last step of the proof accounts for the case when $y \neq 1$, that is, $y=\frac{1-\lambda\left(1-N\left(\frac{\bar{\eta}-1}{\sigma}\right)\right)}{1-N\left(\frac{\bar{\eta}}{\sigma}\right)}$. In 
this case

$$
\begin{aligned}
\frac{\partial y}{\partial \sigma} & =\frac{\partial}{\partial \sigma} \frac{1-\lambda\left(1-N\left(\frac{\bar{\eta}-1}{\sigma}\right)\right)}{1-N\left(\frac{\bar{\eta}}{\sigma}\right)} \\
& =\frac{\lambda n\left(\frac{\bar{\eta}-1}{\sigma}\right)}{1-N\left(\frac{\bar{\eta}}{\sigma}\right)} \frac{1}{\sigma}\left(\frac{\partial \bar{\eta}}{\partial \sigma}-\frac{\bar{\eta}-1}{\sigma}\right)+\frac{1-\lambda\left(1-N\left(\frac{\bar{\eta}-1}{\sigma}\right)\right)}{\left(1-N\left(\frac{\bar{\eta}}{\sigma}\right)\right)^{2}} n\left(\frac{\bar{\eta}}{\sigma}\right) \frac{1}{\sigma}\left(\frac{\partial \bar{\eta}}{\partial \sigma}-\frac{\bar{\eta}}{\sigma}\right) \\
& =-\frac{\lambda n\left(\frac{\bar{\eta}-1}{\sigma}\right)}{1-N\left(\frac{\bar{\eta}}{\sigma}\right)} \frac{1-\pi(\bar{\eta})}{\sigma^{2}}-\frac{1-\lambda\left(1-N\left(\frac{\bar{\eta}-1}{\sigma}\right)\right)}{\left(1-N\left(\frac{\bar{\eta}}{\sigma}\right)\right)^{2}} n\left(\frac{\bar{\eta}}{\sigma}\right) \frac{\pi(\bar{\eta})}{\sigma^{2}}=o(\lambda)
\end{aligned}
$$

because $\pi(\bar{\eta})$ is $o(\lambda)$ implying that

$$
\frac{\partial}{\partial y}\left(\lambda \int_{\bar{\eta} / \sigma}^{\infty} \int_{(\bar{\eta}-1) / \sigma}^{v-1 / \sigma} b^{\prime}(c(u \sigma+1)) n(u) d u(\lambda n(v-1 / \sigma)+y n(v)) d v+A\right) \frac{\partial y}{\partial \sigma}=o\left(\lambda^{2}\right)
$$

so there exists a $\hat{\lambda}$ such that for all $\lambda<\hat{\lambda}$, (56) holds in this case as well.

\section{Proof of Proposition 2}

The expected wage of skilled workers (using Proposition 7) is

$$
\begin{aligned}
\int_{\bar{\eta}} & w(\eta) f_{s}(\eta) d \eta-A F_{s}(\hat{\eta})=\int_{\bar{\eta}}^{\infty} \int_{\bar{\eta}}^{\eta} b(c(v)) \pi^{\prime}(v) f_{s}(\eta) d v d \eta-A \\
& =\int_{\bar{\eta}}^{\infty} \int_{(\bar{\eta}-1) / \sigma}^{(\eta-1) / \sigma} b(c(u \sigma+1)) \sigma \pi^{\prime}(u \sigma+1) f_{s}(\eta) d u d \eta+A \\
& =\int_{(\bar{\eta}-1) / \sigma}^{\infty} \int_{(\bar{\eta}-1) / \sigma}^{v} b(c(u \sigma+1)) \sigma \pi^{\prime}(u \sigma+1) n(v) d u d v+A
\end{aligned}
$$


As before, we first consider the case where $y=1$ so $y$ does not depend on $\sigma$. Taking the derivative of (58) w.r.t. $\sigma$ gives

$$
\begin{aligned}
\frac{d}{d \sigma}\left(\int_{(\bar{\eta}-1) / \sigma}^{\infty} \int_{(\bar{\eta}-1) / \sigma}^{v} b(c(u \sigma+1)) \sigma \pi^{\prime}(u \sigma+1) n(v) d u d v+A\right) \\
=\quad \int_{(\bar{\eta}-1) / \sigma}^{\infty} \int_{(\bar{\eta}-1) / \sigma}^{v} \frac{d}{d \sigma} b(c(u \sigma+1)) \sigma \pi^{\prime}(u \sigma+1) n(v) d u d v \\
\quad+\int_{(\bar{\eta}-1) / \sigma}^{\infty} \int_{(\bar{\eta}-1) / \sigma}^{v} b(c(u \sigma+1)) \frac{d}{d \sigma}\left(\sigma \pi^{\prime}(u \sigma+1)\right) n(v) d u d v \\
=\quad-\int_{(\bar{\eta}-1) / \sigma}^{\infty} \int_{(\bar{\eta}-1) / \sigma}^{v} b^{\prime}(c(u \sigma+1)) \frac{y}{\sigma^{2}} n(u+1 / \sigma) \pi^{\prime}(u \sigma+1) n(v) d u d v \\
\quad-\lambda \int_{(\bar{\eta}-1) / \sigma}^{\infty} \int_{(\bar{\eta}-1) / \sigma}^{v} b(c(u \sigma+1))\left(\frac{y e^{\frac{2 \sigma u+1}{2 \sigma^{2}}}\left(\lambda e^{\frac{2 \sigma u+1}{2 \sigma^{2}}}\left(\sigma^{2}-\sigma u-1\right)+\sigma y(\sigma+u)+y\right)}{\sigma^{4}\left(\lambda e^{\frac{2 \sigma u+1}{2 \sigma^{2}}}+y\right)^{3}}\right) n(v) d u d v \\
<-\lambda \int_{(\bar{\eta}-1) / \sigma}^{\infty} \int_{(\bar{\eta}-1) / \sigma}^{v} b(c(u \sigma+1))\left(\frac{y e^{\frac{2 \sigma u+1}{2 \sigma^{2}}}\left(\lambda e^{\frac{2 \sigma u+1}{2 \sigma^{2}}}\left(\sigma^{2}-\sigma u-1\right)+\sigma y(\sigma+u)+y\right)}{\sigma^{4}\left(\lambda e^{\frac{2 \sigma u+1}{2 \sigma^{2}}}+y\right)^{3}}\right) n(v) d u d v
\end{aligned}
$$

Let $\eta^{*}$ be the negative value of $u$ that solves

$$
\lambda e^{\frac{2 \sigma u+1}{2 \sigma^{2}}}\left(\sigma^{2}-\sigma u-1\right)+\sigma y(\sigma+u)+y=0 .
$$

Notice that if we ignore terms of order $\lambda^{2}$, (59) is less than zero if $\bar{\eta}>\sigma \eta^{*}+1$ which means for small enough $\lambda$, expected wages are decreasing in $\sigma$. So to complete the proof we need to show that for small enough $\lambda$, (59) is also less than zero when

$$
\bar{\eta} \leq \sigma \eta^{*}+1
$$


Switching the order of integration in (59) and then integrating the inner integral gives

$$
\begin{aligned}
& \frac{d}{d \sigma}\left(\int_{(\bar{\eta}-1) / \sigma}^{\infty} \int_{(\bar{\eta}-1) / \sigma}^{v} b(c(u \sigma+1)) \sigma \pi^{\prime}(u \sigma+1) n(v) d u d v+A\right) \\
& <-\lambda \int_{(\bar{\eta}-1) / \sigma}^{\infty} \int_{u}^{\infty} b(c(u \sigma+1))\left(\frac{y e^{\frac{2 \sigma u+1}{2 \sigma^{2}}}\left(\lambda e^{\frac{2 \sigma u+1}{2 \sigma^{2}}}\left(\sigma^{2}-\sigma u-1\right)+\sigma y(\sigma+u)+y\right)}{\sigma^{4}\left(\lambda e^{\frac{2 \sigma u+1}{2 \sigma^{2}}}+y\right)^{3}}\right) n(v) d v d u \\
& =-\lambda \int_{0}^{\infty} b(c(u \sigma+1))\left(\frac{y e^{\frac{2 \sigma u+1}{2 \sigma^{2}}}\left(\lambda e^{\frac{2 \sigma u+1}{2 \sigma^{2}}}\left(\sigma^{2}-\sigma u-1\right)+\sigma y(\sigma+u)+y\right)}{\sigma^{4}\left(\lambda e^{\frac{2 \sigma u+1}{2 \sigma^{2}}}+y\right)^{3}}\right)(1-N(u)) d u \\
& \quad-\lambda \int_{(\bar{\eta}-1) / \sigma}^{\eta^{*}} b(c(u \sigma+1))\left(\frac{y e^{\frac{2 \sigma u+1}{2 \sigma^{2}}}\left(\lambda e^{\frac{2 \sigma u+1}{2 \sigma^{2}}}\left(\sigma^{2}-\sigma u-1\right)+\sigma y(\sigma+u)+y\right)}{\sigma^{4}\left(\lambda e^{\frac{2 \sigma u+1}{2 \sigma^{2}}}+y\right)^{3}}\right)(1-N(u)) d u \\
& \quad-\lambda \int_{\eta^{*}}^{0} b(c(u \sigma+1))\left(\frac{y e^{\frac{2 \sigma u+1}{2 \sigma^{2}}}\left(\lambda e^{\frac{2 \sigma u+1}{2 \sigma^{2}}}\left(\sigma^{2}-\sigma u-1\right)+\sigma y(\sigma+u)+y\right)}{\sigma^{4}\left(\lambda e^{\frac{2 \sigma u+1}{2 \sigma^{2}}}+y\right)^{3}}\right)(1-N(u)) d u
\end{aligned}
$$

where the final step just splits the remaining integral over the three regions. Because $b(\cdot)$ is strictly increasing and $0<N(x) \leq 1 / 2$ in the region $x<0$, we can bound (62) as 
follows:

$$
\begin{aligned}
& \frac{d}{d \sigma}\left(\int_{(\bar{\eta}-1) / \sigma}^{\infty} \int_{(\bar{\eta}-1) / \sigma}^{v} b(c(u \sigma+1)) \sigma \pi^{\prime}(u \sigma+1) n(v) d u d v+A\right) \\
& <-\lambda \int_{0}^{\infty} b(c(u \sigma+1))\left(\frac{y e^{\frac{2 \sigma u+1}{2 \sigma^{2}}}\left(\lambda e^{\frac{2 \sigma u+1}{2 \sigma^{2}}}\left(\sigma^{2}-\sigma u-1\right)+\sigma y(\sigma+u)+y\right)}{\sigma^{4}\left(\lambda e^{\frac{2 \sigma u+1}{2 \sigma^{2}}}+y\right)^{3}}\right)(1-N(u)) d u \\
& -\lambda b\left(c\left(\eta^{*} \sigma+1\right)\right) \int_{(\bar{\eta}-1) / \sigma}^{\eta^{*}}\left(\frac{y e^{\frac{2 \sigma u+1}{2 \sigma^{2}}}\left(\lambda e^{\frac{2 \sigma u+1}{2 \sigma^{2}}}\left(\sigma^{2}-\sigma u-1\right)+\sigma y(\sigma+u)+y\right)}{\sigma^{4}\left(\lambda e^{\frac{2 \sigma u+1}{2 \sigma^{2}}}+y\right)^{3}}\right) d u \\
& -\frac{\lambda}{2} b\left(c\left(\eta^{*} \sigma+1\right)\right) \int_{\eta^{*}}^{0}\left(\frac{y e^{\frac{2 \sigma u+1}{2 \sigma^{2}}}\left(\lambda e^{\frac{2 \sigma u+1}{2 \sigma^{2}}}\left(\sigma^{2}-\sigma u-1\right)+\sigma y(\sigma+u)+y\right)}{\sigma^{4}\left(\lambda e^{\frac{2 \sigma u+1}{2 \sigma^{2}}}+y\right)^{3}}\right) d u \\
& =-\int_{0}^{\infty} b(c(u \sigma+1))\left(\frac{\lambda y e^{\frac{2 \sigma u+1}{2 \sigma^{2}}}\left(\lambda e^{\frac{2 \sigma u+1}{2 \sigma^{2}}}\left(\sigma^{2}-\sigma u-1\right)+\sigma y(\sigma+u)+y\right)}{\sigma^{4}\left(\lambda e^{\frac{2 \sigma u+1}{2 \sigma^{2}}}+y\right)^{3}}\right)(1-N(u)) d u \\
& -\frac{1}{2} b\left(c\left(\eta^{*} \sigma+1\right)\right) \int_{(\bar{\eta}-1) / \sigma}^{0}\left(\frac{\lambda y e^{\frac{2 \sigma u+1}{2 \sigma^{2}}}\left(\lambda e^{\frac{2 \sigma u+1}{2 \sigma^{2}}}\left(\sigma^{2}-\sigma u-1\right)+\sigma y(\sigma+u)+y\right)}{\sigma^{4}\left(\lambda e^{\frac{2 \sigma u+1}{2 \sigma^{2}}}+y\right)^{3}}\right) d u \\
& -\frac{1}{2} b\left(c\left(\eta^{*} \sigma+1\right)\right) \int_{(\bar{\eta}-1) / \sigma}^{\eta^{*}}\left(\frac{\lambda y e^{\frac{2 \sigma u+1}{2 \sigma^{2}}}\left(\lambda e^{\frac{2 \sigma u+1}{2 \sigma^{2}}}\left(\sigma^{2}-\sigma u-1\right)+\sigma y(\sigma+u)+y\right)}{\sigma^{4}\left(\lambda e^{\frac{2 \sigma u+1}{2 \sigma^{2}}}+y\right)^{3}}\right) d u
\end{aligned}
$$

To integrate these integrals, recall that the integrand is

$$
\begin{gathered}
\frac{\lambda y e^{\frac{2 \sigma u+1}{2 \sigma^{2}}}\left(\lambda e^{\frac{2 \sigma u+1}{2 \sigma^{2}}}\left(\sigma^{2}-\sigma u-1\right)+\sigma y(\sigma+u)+y\right)}{\sigma^{4}\left(\lambda e^{\frac{2 \sigma u+1}{2 \sigma^{2}}}+y\right)^{3}} \\
=\frac{d}{d \sigma}\left(\sigma \pi^{\prime}(u \sigma+1)\right)=\pi^{\prime}(u \sigma+1)+\sigma \pi^{\prime \prime}(u \sigma+1) u
\end{gathered}
$$

So integrating by parts gives

$$
\begin{aligned}
\int & \pi^{\prime}(u \sigma+1)+\sigma \pi^{\prime \prime}(u \sigma+1) u d u \\
= & \int \pi^{\prime}(u \sigma+1) d u+\pi^{\prime}(u \sigma+1) u-\int \pi^{\prime}(u \sigma+1) d u \\
= & \pi^{\prime}(u \sigma+1) u
\end{aligned}
$$


Using (65) to integrate the last two expressions in (63) gives

$$
\begin{aligned}
& \frac{d}{d \sigma}\left(\int_{(\bar{\eta}-1) / \sigma}^{\infty} \int_{(\bar{\eta}-1) / \sigma}^{v} b(c(u \sigma+1)) \sigma \pi^{\prime}(u \sigma+1) n(v) d u d v+A\right) \\
& =-\lambda \int_{0}^{\infty} b(c(u \sigma+1))\left(\frac{y e^{\frac{2 \sigma u+1}{2 \sigma^{2}}}\left(\lambda e^{\frac{2 \sigma u+1}{2 \sigma^{2}}}\left(\sigma^{2}-\sigma u-1\right)+\sigma y(\sigma+u)+y\right)}{\sigma^{4}\left(\lambda e^{\frac{2 \sigma u+1}{2 \sigma^{2}}}+y\right)^{3}}\right)(1-N(u)) d u \\
& -\frac{1}{2} b\left(c\left(\eta^{*} \sigma+1\right)\right)\left(\left(\frac{\eta^{*} \lambda y e^{\frac{2 \eta^{*} \sigma+1}{2 \sigma^{2}}}}{\sigma^{2}\left(\lambda e^{\frac{2 \eta^{*} \sigma+1}{2 \sigma^{2}}}+y\right)^{2}}\right)-2\left(\frac{(\bar{\eta}-1) \lambda y e^{\frac{2 \bar{\eta}+1}{2 \sigma^{2}}}}{\sigma^{3}\left(\lambda e^{\frac{\bar{\eta}}{\sigma^{2}}}+e^{\frac{1}{2 \sigma^{2}}} y\right)^{2}}\right)\right) . \\
& =-\lambda \int_{0}^{\infty} b(c(u \sigma+1))\left(\frac{y e^{\frac{2 \sigma u+1}{2 \sigma^{2}}}\left(\lambda e^{\frac{2 \sigma u+1}{2 \sigma^{2}}}\left(\sigma^{2}-\sigma u-1\right)+\sigma y(\sigma+u)+y\right)}{\sigma^{4}\left(\lambda e^{\frac{2 \sigma u+1}{2 \sigma^{2}}}+y\right)^{3}}\right)(1-N(u)) d u \\
& -\frac{\lambda y}{2} b\left(c\left(\eta^{*} \sigma+1\right)\right)\left(\left(\frac{\eta^{*} \sigma e^{\frac{2 \eta^{*} \sigma+1}{2 \sigma^{2}}}}{\sigma^{3}\left(\lambda e^{\frac{2 \eta^{*} \sigma+1}{2 \sigma^{2}}}+y\right)^{2}}\right)-\left(\frac{2(\bar{\eta}-1) e^{\frac{2 \bar{\eta}-1}{2 \sigma^{2}}}}{\sigma^{3}\left(\lambda e^{\frac{2 \bar{\eta}-1}{2 \sigma^{2}}}+y\right)^{2}}\right)\right) .
\end{aligned}
$$

Now using the definition $\eta^{*}$ (that is, (60)) gives

$$
\frac{\eta^{*} \sigma e^{\frac{2 \eta^{*} \sigma+1}{2 \sigma^{2}}}}{\sigma^{3}\left(\lambda e^{\frac{2 \eta^{*} \sigma+1}{2 \sigma^{2}}}+y\right)^{2}}=\frac{-\left(\sigma^{2}+1\right) e^{-1-\frac{1}{2 \sigma^{2}}}}{\sigma^{3}\left(\lambda e^{\frac{2 \eta^{*} \sigma+1}{2 \sigma^{2}}}+y\right)^{2}}+o\left(\lambda^{2}\right)
$$


Substituting this expression into (66) and separating out terms of $\lambda^{2}$ or greater gives

$$
\begin{aligned}
& \frac{d}{d \sigma}\left(\int_{(\bar{\eta}-1) / \sigma}^{\infty} \int_{(\bar{\eta}-1) / \sigma}^{v} b(c(u \sigma+1)) \sigma \pi^{\prime}(u \sigma+1) n(v) d u d v+A\right) \\
& <-\lambda \int_{0}^{\infty} b(c(u \sigma+1))\left(\frac{e^{\frac{2 \sigma u+1}{2 \sigma^{2}}}(\sigma(2 \sigma+u)+1)}{\sigma^{4}\left(\lambda e^{\frac{2 \sigma u+1}{2 \sigma^{2}}}+1\right)^{3}}\right)(1-N(u)) d u \\
& \left.\quad-\frac{\lambda y}{2} b\left(c\left(\eta^{*} \sigma+1\right)\right)\left(\frac{-\left(\sigma^{2}+1\right) e^{-1-\frac{1}{2 \sigma^{2}}}}{\sigma^{3}\left(\lambda e^{\frac{2 \eta^{*} \sigma+1}{2 \sigma^{2}}}+y\right)^{2}}\right)-\left(\frac{2(\bar{\eta}-1) e^{\frac{2 \bar{\eta}-1}{2 \sigma^{2}}}}{\sigma^{3}\left(\lambda e^{\frac{2 \bar{\eta}-1}{2 \sigma^{2}}}+y\right)^{2}}\right)\right)+o\left(\lambda^{2}\right) \\
& \left.<-\frac{\lambda y}{2} b\left(c\left(\eta^{*} \sigma+1\right)\right)\left(\frac{-\left(\sigma^{2}+1\right) e^{-1-\frac{1}{2 \sigma^{2}}}}{\sigma^{3}\left(\lambda e^{\frac{2 \eta^{*} \sigma+1}{2 \sigma^{2}}}+y\right)^{2}}\right)-\left(\frac{-2\left(\sigma^{2}+1\right) e^{-1-\frac{1}{2 \sigma^{2}}}}{\sigma^{3}\left(\lambda e^{\frac{2 \bar{\eta}-1}{2 \sigma^{2}}}+y\right)^{2}}\right)\right)+o\left(\lambda^{2}\right) \\
& =\quad-\frac{\lambda y}{2} b\left(c\left(\eta^{*} \sigma+1\right)\right)\left(\frac{\left(\sigma^{2}+1\right) e^{-1-\frac{1}{2 \sigma^{2}}}}{\sigma^{3}\left(\lambda e^{\frac{2 \eta^{*} \sigma+1}{2 \sigma^{2}}}+y\right)^{2}\left(\lambda e^{\frac{2 \bar{\eta}-1}{2 \sigma^{2}}}+y\right)^{2}}\right)+o\left(\lambda^{2}\right) \\
& <0
\end{aligned}
$$

where the last inequality follows because $\bar{\eta}$ is bounded by (61) so $\bar{\eta} \leq-\sigma^{2}$. All that now

remains is to show that this is also true when $y \neq 1$. Notice from (58) that $y$ only enters the expected wage function through the function $\pi^{\prime}(\cdot)$. Now

$$
\frac{\partial \pi^{\prime}(x)}{\partial y} \frac{\partial y}{\partial \sigma}=\left(\frac{\lambda^{2} e^{\frac{4 x+1}{2 \sigma^{2}}}-\lambda y e^{\frac{x+1}{\sigma^{2}}}}{\sigma^{2}\left(\lambda e^{\frac{x}{\sigma^{2}}}+e^{\frac{1}{2 \sigma^{2}}} y\right)^{3}}\right) \frac{\partial y}{\partial \sigma}=o\left(\lambda^{2}\right)
$$

because, from (57), $\frac{\partial y}{\partial \sigma}$ is order $\lambda$. So there exists a $\hat{\lambda}$ such that for all $\lambda<\hat{\lambda}$ where an equilibrium exists,

$$
\frac{d}{d \sigma}\left(\int_{(\bar{\eta}-1) / \sigma}^{\infty} \int_{(\bar{\eta}-1) / \sigma}^{v} b(c(u \sigma+1)) \sigma \pi^{\prime}(u \sigma+1) n(v) d u d v+A\right)<0 .
$$

\section{E Proof of Proposition 3}

Overall welfare is defined to be

$$
\left(1-F_{s}(\bar{\eta})\right)-A\left(F_{s}(\bar{\eta})+F_{u}(\bar{\eta})\right)=1-N\left(\frac{\bar{\eta}-1}{\sigma}\right)-A\left(N\left(\frac{\bar{\eta}-1}{\sigma}\right)+N\left(\frac{\bar{\eta}}{\sigma}\right)\right)
$$


Beginning with the case when $y=1$ and taking the derivative w.r.t. $\sigma$ and using the expression for $\frac{\partial \bar{\eta}}{\partial \sigma}$ derived in the proof of Proposition 1 gives

$$
\begin{aligned}
& \frac{d}{d \sigma}\left(\left(1-N\left(\frac{\bar{\eta}-1}{\sigma}\right)\right)-A\left(N\left(\frac{\bar{\eta}-1}{\sigma}\right)+N\left(\frac{\bar{\eta}}{\sigma}\right)\right)\right) \\
& \quad=-n\left(\frac{\bar{\eta}-1}{\sigma}\right)\left(\frac{\partial \bar{\eta}}{\partial \sigma}-\frac{\bar{\eta}-1}{\sigma}\right)-A\left(n\left(\frac{\bar{\eta}-1}{\sigma}\right)\left(\frac{\partial \bar{\eta}}{\partial \sigma}-\frac{\bar{\eta}-1}{\sigma}\right)+n\left(\frac{\bar{\eta}}{\sigma}\right)\left(\frac{\partial \bar{\eta}}{\partial \sigma}-\frac{\bar{\eta}}{\sigma}\right)\right) \\
& =-n\left(\frac{\bar{\eta}-1}{\sigma}\right)\left(\frac{1-\pi(\bar{\eta})}{\sigma}\right)-A\left(n\left(\frac{\bar{\eta}-1}{\sigma}\right)\left(\frac{1-\pi(\bar{\eta})}{\sigma}\right)-n\left(\frac{\bar{\eta}}{\sigma}\right)\left(\frac{\pi(\bar{\eta})}{\sigma}\right)\right) \\
& =-\left(\frac{n\left(\frac{\bar{\eta}-1}{\sigma}\right) n\left(\frac{\bar{\eta}}{\sigma}\right)}{\sigma\left(\lambda n\left(\frac{\bar{\eta}-1}{\sigma}\right)+y n\left(\frac{\bar{\eta}}{\sigma}\right)\right)}\right)(y+A y-A \lambda) \\
& <0 .
\end{aligned}
$$

The last step of the proof accounts for the case when $y \neq 1$. In this case we have already seen that

$$
\frac{\partial y}{\partial \sigma}=o(\lambda)
$$

implying (using (51)) that

$$
\frac{d \bar{\eta}}{d \sigma}=\frac{\partial \bar{\eta}}{\partial \sigma}+\frac{1-F_{u}}{\lambda f_{s}(\bar{\eta})+y f_{u}(\bar{\eta})} \frac{\partial y}{\partial \sigma}=\frac{\partial \bar{\eta}}{\partial \sigma}+o(\lambda)
$$

so there exists a $\hat{\lambda}$ such that for all equilibria that feature $\lambda<\hat{\lambda}$, (68) holds in this case as well.

\section{F Proof of Proposition 4}

In the equilibrium without a standard, consumer surplus is

$$
\int_{\bar{\eta}}^{\infty} b(c(\eta)) \pi(\eta)-w(\eta) d c(\eta)=\lambda \int_{\bar{\eta}}^{\infty} \int_{\bar{\eta}}^{\eta} b^{\prime}(c(v)) f_{s}(v) d v d c(\eta)+A
$$

where we have used (10). Next recall that if an equilibrium exists then the expected benefit of unskilled agents to produce must be non-negative. Applying this condition to (29) implies:

$$
\int_{\bar{\eta}}^{\infty} \int_{\bar{\eta}}^{\eta} b(c(v)) \pi^{\prime}(v) f_{u}(\eta) d v d \eta \geq A
$$


Now consider introducing into this equilibrium a minimum standard $\hat{\eta}=\bar{\eta}$. Note that an equilibrium will always exists because (42) is satisfied whenever (16) is satisfied. When $y<$ 1 in the equilibrium without a standard, because (18) and (43) are the same, introducing a minimum standard leaves $y$ unchanged. When $y=1$, from Proposition 8 , in this equilibrium the expected benefit of producing for an unskilled agent is

$$
\begin{aligned}
\int_{\bar{\eta}}^{\infty} \int_{\bar{\eta}}^{\eta} b\left(\lambda F_{s}(v)+F_{u}(v)-\lambda\right) \pi^{\prime}(v) f_{u}(\eta) d v d \eta+ \\
b\left(\lambda F_{s}(\bar{\eta})+F_{u}(\bar{\eta})-\lambda\right) \pi(\bar{\eta})\left(1-F_{u}(\bar{\eta})\right)-A F_{u}(\bar{\eta}) \\
\geq A+b\left(\lambda F_{s}(\bar{\eta})+F_{u}(\bar{\eta})-\lambda\right) \pi(\bar{\eta})\left(1-F_{u}(\bar{\eta})\right)-A F_{u}(\bar{\eta}) \\
=A\left(1-F_{u}(\bar{\eta})\right)+b\left(\lambda F_{s}(\bar{\eta})+F_{u}(\bar{\eta})-\lambda\right) \pi(\bar{\eta})\left(1-F_{u}(\bar{\eta})\right) \geq 0,
\end{aligned}
$$

where the inequality follows from (70). What this implies that if a minimum standard $\hat{\eta}=\bar{\eta}$ is introduced into an equilibrium without a standard that features $y=1$, the resulting equilibrium features $y=1$. So $c(\eta)$ is identical in the two equilibria.

When $y=1$, consumer surplus in the equilibrium with a minimum standard is

$$
\int_{\hat{\eta}}^{\infty} b(c(\eta)) \pi(\eta)-w(\eta) d c(\eta)=\lambda \int_{\hat{\eta}}^{\infty} \int_{\hat{\eta}}^{\eta} b^{\prime}(c(v)) f_{s}(v) c^{\prime}(\eta) d v d \eta
$$

where we have used (1), (30) and (31). Subtracting (69) from (71) when $\hat{\eta}=\bar{\eta}$ gives

$$
\begin{aligned}
\lambda \int_{\bar{\eta}}^{\infty} \int_{\bar{\eta}}^{\eta} b^{\prime}(c(v)) f_{s}(v) c^{\prime}(\eta) d v d \eta & -\left(\lambda \int_{\bar{\eta}}^{\infty} \int_{\bar{\eta}}^{\eta} b^{\prime}(c(v)) f_{s}(v) c^{\prime}(\eta) d v d \eta+A\right) \\
& =-A \leq 0
\end{aligned}
$$

so introducing a minimum standard $\hat{\eta}=\bar{\eta}$ cannot increase consumer surplus. Obviously, introducing a standard below $\bar{\eta}$ has no effect, so we have shown that a government cannot increase consumer surplus by introducing a standard $\hat{\eta} \leq \bar{\eta}$.

For minimum standards $\hat{\eta} \geq \bar{\eta} \geq \Delta$ the slope of consumer surplus is

$$
\begin{gathered}
\frac{d}{d \hat{\eta}} \int_{\hat{\eta}}^{\infty} b(c(\eta)) \pi(\eta)-w(\eta) d c(\eta)=\frac{d}{d \hat{\eta}} \int_{\hat{\eta}}^{\infty} \int_{\hat{\eta}}^{\eta} b^{\prime}(c(v)) \lambda f_{s}(v) d u c^{\prime}(\eta) d \eta \\
=-b^{\prime}(c(\hat{\eta})) \lambda f_{s}(\hat{\eta})(1-c(\hat{\eta}))+\int_{\hat{\eta}}^{\infty} \int_{\hat{\eta}}^{\eta} b^{\prime}(c(v)) \lambda f_{s}(v) f_{u}(\eta) \frac{d y}{d \hat{\eta}} d v d \eta \\
\quad-\int_{\hat{\eta}}^{\infty} \int_{\hat{\eta}}^{\eta} b^{\prime \prime}(c(v))\left(1-F_{u}(\bar{\eta})\right) \frac{d y}{d \hat{\eta}} \lambda f_{s}(v)\left(\lambda f_{s}(\eta)+y f_{u}(\eta)\right) d v d \eta
\end{gathered}
$$


Notice that $b^{\prime \prime}(\cdot) \leq 0$ and $\frac{d y}{d \hat{\eta}}=0$ over the intervals where $y=1$ or $y=0$. When $0 \leq y \leq 1$, by Lemma 1 , there exists a $\lambda$ small enough so that $\frac{d y}{d \hat{\eta}}<0$. These conditions jointly imply that for a small enough $\lambda$, (72) is strictly less than zero. So $\hat{\eta} \geq \bar{\eta}$ leads to strictly lower consumer surplus when $\bar{\eta} \geq \Delta$, that is, $y=1$ in the equilibrium without a minimum standard.

In the case when $\bar{\eta}<\Delta$ (that is, $y<1$ in the equilibrium without a minimum standard), consumer surplus in the equilibrium with a minimum standard is

$$
\begin{aligned}
\int_{\hat{\eta}}^{\infty} b(c(\eta)) \pi(\eta) & -w(\eta) d c(\eta)=\lambda \int_{\hat{\eta}}^{\infty} \int_{\hat{\eta}}^{\eta} b^{\prime}(c(v)) f_{s}(v) c^{\prime}(\eta) d v d \eta \\
& +\left(\frac{1}{1-F_{u}(\hat{\eta})}\right) \int_{\hat{\eta}}^{\infty} \int_{\hat{\eta}}^{x} b(c(v)) \pi^{\prime}(v) d v f_{u}(x) d x-A\left(\frac{F_{u}(\hat{\eta})}{1-F_{u}(\hat{\eta})}\right) .
\end{aligned}
$$

Subtracting (69) from (73) when $\hat{\eta}=\bar{\eta}$ gives

$$
\begin{aligned}
& \left(\frac{1}{1-F_{u}(\bar{\eta})}\right) \int_{\bar{\eta}}^{\infty} \int_{\bar{\eta}}^{x} b(c(v)) \pi^{\prime}(v) d v f_{u}(x) d x-A\left(\frac{F_{u}(\bar{\eta})}{1-F_{u}(\bar{\eta})}\right)-A \\
& =\left(\frac{1}{1-F_{u}(\bar{\eta})}\right)\left(\int_{\bar{\eta}}^{\infty} \int_{\bar{\eta}}^{x} b(c(v)) \pi^{\prime}(v) d v f_{u}(x) d x-A\right)=0
\end{aligned}
$$

because $y$ is the same in both equilibria and so (19) holds. So consumer surplus remains the same. Finally, we need to show that consumer surplus is decreasing in $\hat{\eta}$, when $y<1$.

$$
\begin{aligned}
\frac{d}{d \hat{\eta}} \int_{\hat{\eta}}^{\infty} b(c(\eta)) \pi(\eta)-w(\eta) d c(\eta)= & w(\hat{\eta}) c^{\prime}(\hat{\eta})+\int_{\hat{\eta}}^{\infty} b^{\prime}(c(\eta)) c_{\hat{\eta}}(\eta) \pi(\eta) d c(\eta)+\int_{\hat{\eta}}^{\infty} b(c(\eta)) \pi_{\hat{\eta}}(\eta) d c(\eta) \\
& -\int_{\hat{\eta}}^{\infty} w_{\hat{\eta}}(\eta) d c(\eta)+\int_{\hat{\eta}}^{\infty}(b(c(\eta)) \pi(\eta)-w(\eta)) f_{u}(\eta) \frac{d y}{d \hat{\eta}} d \eta \\
= & w(\hat{\eta}) c^{\prime}(\eta)+\int_{\hat{\eta}}^{\infty} b^{\prime}(c(\eta)) c_{\hat{\eta}}(\eta) \pi(\eta) d c(\eta) \\
& -\int_{\hat{\eta}}^{\infty} w_{\hat{\eta}}(\eta) d c(\eta)-\frac{d y}{d \hat{\eta}} \int_{\hat{\eta}}^{\infty} w(\eta) f_{u}(\eta) d \eta \\
= & w(\hat{\eta}) c^{\prime}(\eta)+\int_{\hat{\eta}}^{\infty} b^{\prime}(c(\eta)) c_{\hat{\eta}}(\eta) \pi(\eta) d c(\eta) \\
& -\int_{\hat{\eta}}^{\infty} w_{\hat{\eta}}(\eta) d c(\eta)-\frac{d y}{d \hat{\eta}} A F_{u}(\hat{\eta})
\end{aligned}
$$


where the second equality follows because

$$
\begin{aligned}
\int_{\hat{\eta}}^{\infty} b(c(\eta)) \pi_{\hat{\eta}}(\eta) d c(\eta) & =-\frac{d y}{d \hat{\eta}} \int_{\hat{\eta}}^{\infty} b(c(\eta)) \pi(\eta) \frac{f_{u}(\eta)}{\lambda f_{s}(\eta)+y f_{u}(\eta)} d c(\eta) \\
& =-\frac{d y}{d \hat{\eta}} \int_{\hat{\eta}}^{\infty} b(c(\eta)) \pi(\eta) \frac{f_{u}(\eta)}{\lambda f_{s}(\eta)+y f_{u}(\eta)}\left(\lambda f_{s}(\eta)+y f_{u}(\eta)\right) d \eta \\
& =-\frac{d y}{d \hat{\eta}} \int_{\hat{\eta}}^{\infty} b(c(\eta)) \pi(\eta) f_{u}(\eta) d \eta
\end{aligned}
$$

and the last line follows from (40). Now the first term of $(74)$ is negative because $w(\hat{\eta})<0$, and the second and last terms are less than or equal to zero because

$$
\frac{d y}{d \hat{\eta}}=\frac{\lambda f_{s}(\hat{\eta})+y f_{u}(\hat{\eta})}{1-F_{u}(\hat{\eta})}>0
$$

from (43) and

$$
c_{\hat{\eta}}(\eta)=\left(F_{u}(\eta)-1\right) \frac{d y}{d \hat{\eta}} .
$$

So we need to show the third term of (74) is less than or equal to zero. Now

$$
\begin{aligned}
\int_{\hat{\eta}}^{\infty} w_{\hat{\eta}}(\eta) d c(\eta) & =\int_{\hat{\eta}}^{\infty} w_{\hat{\eta}}(\eta)\left(\lambda f_{s}(\eta)+y f_{u}(\eta)\right) d \eta \\
& \geq y A f_{u}(\hat{\eta})+y w(\hat{\eta}) f_{u}(\hat{\eta})+\frac{A f_{u}(\hat{\eta})+w(\hat{\eta}) f_{u}(\hat{\eta})}{1-F_{u}(\hat{\eta})}\left(1-F_{s}(\hat{\eta})\right) \lambda
\end{aligned}
$$

because taking derivatives in (40) and rearranging gives

$$
\int_{\hat{\eta}}^{\infty} w_{\hat{\eta}}(\eta) f_{u}(\eta) d \eta=A f_{u}(\hat{\eta})+w(\hat{\eta}) f_{u}(\hat{\eta})
$$

and then

$$
\begin{gathered}
\int_{\hat{\eta}}^{\infty}\left(w_{\hat{\eta}}(\eta)-\frac{A f_{u}(\hat{\eta})+w(\hat{\eta}) f_{u}(\hat{\eta})}{1-F_{u}(\hat{\eta})}\right) f_{u}(\eta) d \eta=0 \\
\int_{\hat{\eta}}^{\infty}\left(w_{\hat{\eta}}(\eta)-\frac{A f_{u}(\hat{\eta})+w(\hat{\eta}) f_{u}(\hat{\eta})}{1-F_{u}(\hat{\eta})}\right) f_{u}(\eta) \frac{f_{s}(\eta)}{f_{u}(\eta)} d \eta \geq 0
\end{gathered}
$$

because $\frac{f_{s}(\eta)}{f_{u}(\eta)}$ is increasing which means

$$
\int_{\hat{\eta}}^{\infty}\left(w_{\hat{\eta}}(\eta)-\frac{A f_{u}(\hat{\eta})+w(\hat{\eta}) f_{u}(\hat{\eta})}{1-F_{u}(\hat{\eta})}\right) f_{s}(\eta) d \eta \geq 0
$$


SO

$$
\int_{\hat{\eta}}^{\infty} w_{\hat{\eta}}(\eta) f_{s}(\eta) d \eta \geq \frac{A f_{u}(\hat{\eta})+w(\hat{\eta}) f_{u}(\hat{\eta})}{1-F_{u}(\hat{\eta})}\left(1-F_{s}(\hat{\eta})\right)
$$

Rewriting (75)

$$
\begin{aligned}
\int_{\hat{\eta}}^{\infty} w_{\hat{\eta}}(\eta) d c(\eta) & \geq y A f_{u}(\hat{\eta})+y w(\hat{\eta}) f_{u}(\hat{\eta})+\frac{A f_{u}(\hat{\eta})+w(\hat{\eta}) f_{u}(\hat{\eta})}{1-F_{u}(\hat{\eta})}\left(1-F_{s}(\hat{\eta})\right) \lambda \\
& =y f_{u}(\hat{\eta})(A+w(\hat{\eta}))\left(1+\frac{1-F_{s}(\hat{\eta})}{1-F_{u}(\hat{\eta})}\right)(\lambda) \\
& \geq 0
\end{aligned}
$$

because $A \geq-w(\hat{\eta})$ because producers choose to produce when $\eta=\hat{\eta}$. Thus we have shown that

$$
\frac{d}{d \hat{\eta}} \int_{\hat{\eta}}^{\infty} b(c(\eta)) \pi(\eta)-w(\eta) d c(\eta)<0
$$

which implies that $\hat{\eta}>\bar{\eta}$ leads to strictly lower consumer surplus in the case when $\bar{\eta}<\Delta$.

\section{G Proof of Proposition 5}

To prove the proposition we need to find a standard such that expect wages of skilled workers are strictly larger under this standard than they are in the equilibrium without the standard. Wages in the equilibrium without a minimum standard are, by Proposition 7 ,

$$
w(\eta)=\int_{\bar{\eta}}^{\eta} b(c(v)) \pi^{\prime}(v) d u-A
$$

If a standard is introduced into this equilibrium such that $\hat{\eta}=\bar{\eta}$, as we saw in the proof of Proposition $4, c(\eta)$ and $y$ are identical in the two equilibria. When $y=1$ in the equilibrium without a minimum standard, by Proposition 8 , wages when the standard is introduced are

$$
w(\eta)=\int_{\bar{\eta}}^{\eta} b(c(v)) \pi^{\prime}(v) d u+b(c(\bar{\eta})) \pi(\bar{\eta})=\int_{\bar{\eta}}^{\eta} b(c(v)) \pi^{\prime}(v) d u
$$

because $c(\bar{\eta})=0$. Subtracting (76) from (77) shows that wages always increase by $A$. That implies that expected wages increase by $A\left(1-F_{s}(\bar{\eta})\right)$. So, when $A>0$, we have shown that expected wages are strictly higher when the standard is introduced. To show that wages strictly increase in all cases, we show that the derivative of the expected wage of skilled workers is positive when $A=0$ and $\hat{\eta}=\bar{\eta}$, so by marginally raising the 
standard above $\bar{\eta}$ expected wages can be made strictly higher in this case. Calculating the derivative of expected wages when $A=0$ (using (15) and noting from Proposition 7 that when $A=0, y=1$ for all $\hat{\eta})$ :

$$
\begin{aligned}
& \frac{d}{d \hat{\eta}}\left(\int_{\bar{\eta}}^{\infty} w(\eta) f_{s}(\eta) d \eta\right) \\
& \quad=\frac{d}{d \hat{\eta}}\left(\int_{\bar{\eta}}^{\infty} b(c(\eta)) \pi(\eta) f_{s}(\eta) d \eta-\lambda \int_{\bar{\eta}}^{\infty} \int_{\hat{\eta}}^{\eta} b^{\prime}(c(v)) f_{s}(v) f_{s}(\eta) d u d \eta\right) \\
& =-b(c(\bar{\eta})) \pi(\bar{\eta}) f_{s}(\bar{\eta})+\lambda b^{\prime}(c(\bar{\eta})) f_{s}(\bar{\eta})\left(1-F_{s}(\bar{\eta})\right) \\
& =\lambda b^{\prime}(c(\bar{\eta})) f_{s}(\bar{\eta})\left(1-F_{s}(\bar{\eta})\right)>0 .
\end{aligned}
$$

because $c(\bar{\eta})=0$ and so $b(c(\bar{\eta}))=0$.

Finally, we have to consider the case when $\bar{\eta}<\Delta$. In this case $y<1$ so an equilibrium can only exist with $A>0$. As above, introduce a minimum standard such that $\hat{\eta}=\bar{\eta}$. By Proposition 9, wages when this standard is introduced (using (19)) are

$$
\begin{aligned}
w(\eta) & =\int_{\bar{\eta}}^{\eta} b(c(v)) \pi^{\prime}(v) d u-\left(\frac{1}{1-F_{u}(\bar{\eta})}\right) \int_{\bar{\eta}}^{\infty} \int_{\bar{\eta}}^{x} b(c(v)) \pi^{\prime}(v) d v f_{u}(x) d x+A\left(\frac{F_{u}(\bar{\eta})}{1-F_{u}(\bar{\eta})}\right) \\
& =\int_{\bar{\eta}}^{\eta} b(c(v)) \pi^{\prime}(v) d u-\left(\frac{A}{1-F_{u}(\bar{\eta})}\right)+A\left(\frac{F_{u}(\bar{\eta})}{1-F_{u}(\bar{\eta})}\right) \\
& =\int_{\bar{\eta}}^{\eta} b(c(v)) \pi^{\prime}(v) d u-A
\end{aligned}
$$

which is (76), so introducing the standard leaves skilled workers indifferent. Now consider the effect of raising the standard to $\Delta$. Now wages are given by (77), although because $y$ differs in the two standards, the integrand of (77) is not the same as the integrand of (78). Because the maximum difference between the two values of $y$ is $\lambda$, the overall difference is at least of order $\lambda$, so the wages with a standard of $\Delta$ exceed wages with a standard of $\bar{\eta}$ by $A+o(\lambda)$. Over the region $\bar{\eta} \leq \eta \leq \Delta$ wages with a standard of $\Delta$ exceed wages with a standard of $\bar{\eta}$ by $(-w(\eta)-A)$. So expected wages with a standard of $\Delta$ exceed 
expected wages with a standard of $\bar{\eta}$ by

$$
\begin{aligned}
A & \left(1-F_{s}(\Delta)\right)+\int_{\bar{\eta}}^{\Delta}(-w(\eta)-A) f_{s}(\eta) d \eta+o(\lambda) \\
& =A\left(1-F_{s}(\Delta)\right)-A\left(F_{s}(\Delta)-F_{s}(\bar{\eta})\right)-\int_{\bar{\eta}}^{\Delta} w(\eta) f_{s}(\eta) d \eta+o(\lambda) \\
& =A\left(1-F_{s}(\Delta)\right)-A\left(F_{s}(\Delta)-F_{s}(\bar{\eta})\right)+A\left(F_{s}(\Delta)-F_{s}(\bar{\eta})\right)+o(\lambda) \\
& =A\left(1-F_{s}(\Delta)\right)+o(\lambda)
\end{aligned}
$$

where $w(\eta)$ is the wage function with a standard of $\bar{\eta}$, that is, (78). So there exists a $\lambda^{*}$ such that for all $\lambda<\lambda^{*}$ where an equilibrium exists, expected wages are strictly larger when a standard of $\Delta$ is introduced.

\section{H Proof of Proposition 6}

Overall welfare is defined to be

$$
\left(1-F_{s}(\bar{\eta})\right)-A\left(F_{s}(\bar{\eta})+F_{u}(\bar{\eta})\right)
$$

Setting $\hat{\eta}=\bar{\eta}$ and taking the derivative w.r.t. $\hat{\eta}$ gives

$$
\left.-f_{s}(\hat{\eta})\right)-A\left(f_{s}(\hat{\eta})+f_{u}(\hat{\eta})\right)<0
$$

\section{Lemmas}

In a equilibrium of the economy where a minimum standard $\hat{\eta} \geq \Delta$ is imposed, define $\Gamma$ to be the infinum of $\hat{\eta}$ such that $y<1$ (the largest value of $\hat{\eta}$ that is smaller than or equal to any value of $\hat{\eta}$ when $y<1$ ).

Lemma 1 In a equilibrium of the economy where a minimum standard $\hat{\eta} \geq \Gamma$ is imposed and the equilibrium features $0<y<1$, then for any there exists $a \lambda^{*}>0$ such that for all $\lambda<\lambda^{*}$ :

$$
\lambda b^{\prime}(c(\hat{\eta}))\left(1-F_{u}(\hat{\eta})\right) \frac{f_{s}(\hat{\eta})}{f_{u}(\hat{\eta})}-w(\hat{\eta})-A<0
$$

and

$$
\frac{d y}{d \hat{\eta}}<0 .
$$


Proof. Notice that if $A=0$, then $y=1$ for all $\hat{\eta}$ because it is impossible for unskilled expected wages to equal zero implying that $A>0$. Then the first condition follows trivially from the fact that the first term in (79) and $w(\hat{\eta})$ are order $\lambda$. When $0<y<1$, expected unskilled wages are always zero, so taking the derivative of expected the expected wage w.r.t. $\hat{\eta}$ gives

$$
-A f_{u}(\hat{\eta})+\int_{\hat{\eta}}^{\infty} w_{\hat{\eta}}(\eta) f_{u}(\eta) d \eta-w(\hat{\eta}) f_{u}(\hat{\eta})=0 .
$$

Now,

$$
\begin{aligned}
w_{\hat{\eta}}(\eta)= & \lambda b^{\prime}(c(\hat{\eta})) f_{s}(\hat{\eta})-\lambda \int_{\hat{\eta}}^{\eta} b^{\prime \prime}(c(v))\left(F_{u}(u)-1\right) f_{s}(\eta) \frac{d y}{d \hat{\eta}} d v \\
& +b^{\prime}(c(\eta))\left(F_{u}(\eta)-1\right) \pi(\eta) \frac{d y}{d \hat{\eta}}+b(c(\eta)) \pi_{y}(\eta) \frac{d y}{d \hat{\eta}}
\end{aligned}
$$

Substituting gives

$$
\begin{aligned}
& -\left(b(c(\hat{\eta})) \pi(\hat{\eta})-\lambda b^{\prime}(c(\hat{\eta}))\left(1-F_{u}(\hat{\eta})\right) \frac{f_{s}(\hat{\eta})}{f_{u}(\hat{\eta})}+A\right) f_{u}(\hat{\eta}) \\
& +\int_{\hat{\eta}}^{\infty} b^{\prime}(c(\eta))\left(F_{u}(\eta)-1\right) \pi(\eta) f_{u}(\eta) \frac{d y}{d \hat{\eta}} d \eta+\int_{\hat{\eta}}^{\infty} b(c(\eta)) \pi_{y}(\eta) f_{u}(\eta) \frac{d y}{d \hat{\eta}} d \eta \\
& -\lambda \int_{\hat{\eta}}^{\infty} \int_{\hat{\eta}}^{\eta} b^{\prime \prime}(c(v))\left(F_{u}(u)-1\right) f_{s}(v) f_{u}(\eta) \frac{d y}{d \hat{\eta}} d v d \eta=0
\end{aligned}
$$

implying

$$
\begin{aligned}
& \left(b(c(\hat{\eta})) \pi(\hat{\eta})-\lambda b^{\prime}(c(\hat{\eta}))\left(1-F_{u}(\hat{\eta})\right) \frac{f_{s}(\hat{\eta})}{f_{u}(\hat{\eta})}+A\right) f_{u}(\hat{\eta})= \\
& \left(\int_{\hat{\eta}}^{\infty} b^{\prime}(c(\eta))\left(F_{u}(u)-1\right) \pi(\eta) f_{u}(\eta) d \eta+\int_{\hat{\eta}}^{\infty} b(c(\eta)) \pi_{y}(\eta) f_{u}(\eta) d \eta\right. \\
& \left.-\lambda \int_{\hat{\eta}}^{\infty} \int_{\hat{\eta}}^{\eta} b^{\prime \prime}(c(v))\left(F_{u}(u)-1\right) f_{s}(v) f_{u}(\eta) d v d \eta\right) \frac{d y}{d \hat{\eta}}
\end{aligned}
$$

so we have

$$
\begin{gathered}
\frac{d y}{d \hat{\eta}}= \\
\frac{\left(b(c(\hat{\eta})) \pi(\hat{\eta})-\lambda b^{\prime}(c(\hat{\eta}))\left(1-F_{u}(\hat{\eta})\right) \frac{f_{s}(\hat{\eta})}{f_{u}(\hat{\eta})}+A\right) f_{u}(\hat{\eta})}{\int_{\hat{\eta}}^{\infty}\left(b(c(\eta)) \pi_{y}(\eta)-b^{\prime}(c(\eta))\left(1-F_{u}(u)\right) \pi(\eta)+\lambda \int_{\hat{\eta}}^{\eta} b^{\prime \prime}(c(v))\left(1-F_{u}(u)\right) f_{s}(v) d v\right) f_{u}(\eta) d \eta}
\end{gathered}
$$


The denominator is negative because all the terms in the integrands are negative because $b^{\prime \prime}(c(v)) \leq 0$ and $\pi_{y}(\eta)<0$. The numerator converges to A for $\lambda$ small enough. Thus for small enough $\lambda$, (81) is negative. 